\title{
Time-Delay Luenberger Observer Design for Sliding Mode Control of Nonlinear Markovian Jump Systems via Event-Triggered Mechanism
}

\author{
Min Cheng ${ }^{1}$, Chunyang Zhang ${ }^{2}$, Jin Qiu ${ }^{3}$, Zhengtian $\mathrm{Wu}^{3}{ }^{\circledR}$ and Qing Gao ${ }^{2,4, *}$ \\ 1 School of Mechatronic Engineering and Automation, Shanghai University, Shanghai 200444, China; \\ abcheng@shu.edu.cn \\ 2 School of Automation Science and Electrical Engineering, Beihang University, Beijing 100191, China; \\ chunyangzhang@buaa.edu.cn \\ 3 School of Electronic and Information Engineering, Suzhou University of Science and Technology, \\ Suzhou 215009, China; kyky11@mail.usts.edu.cn (J.Q.); wzht8@mail.usts.edu.cn (Z.W.) \\ 4 Institute for Automatic Control and Complex Systems (AKS), University of Duisburg-Essen, \\ 47057 Duisburg, Germany \\ * Correspondence: gaoqing@buaa.edu.cn
}

check for updates

Citation: Cheng, M.; Zhang, C.; Qiu, J.; Wu, Z.; Gao, Q. Time-Delay Luenberger Observer Design for Sliding Mode Control of Nonlinear Markovian Jump Systems via Event-Triggered Mechanism. Machines 2021, 9, 259. https:// doi.org/10.3390/machines9110259

Academic Editors:

Christoph M. Hackl and Hai Wang

Received: 7 August 2021

Accepted: 27 October 2021

Published: 30 October 2021

Publisher's Note: MDPI stays neutral with regard to jurisdictional claims in published maps and institutional affiliations.

Copyright: (c) 2021 by the authors. Licensee MDPI, Basel, Switzerland. This article is an open access article distributed under the terms and conditions of the Creative Commons Attribution (CC BY) license (https:/ / creativecommons.org/licenses/by/ $4.0 /)$.

\begin{abstract}
This paper is focused on the stabilization of Takagi-Sugeno fuzzy model-based Markovian jump systems with the aid of a delayed state observer. Due to network-induced constraints in the communication channel, a delay partition method combined with an event-triggered mechanism is proposed to design the observer. Then, a novel integral sliding surface is designed, based on which sliding mode dynamics is obtained. Further, according to stochastic stability theory, feasible conditions are provided to ensure the sliding mode dynamics and the error dynamics have an $H_{\infty}$ attenuate level $\gamma$. The challenge is to deal with the issue that transition rates may be totally unknown. Moreover, an observer-based sliding mode controller is constructed to ensure the finitetime reachability of the predefined sliding surface. Finally, a numerical example based on a robotic manipulator is given to verify the effectiveness of the proposed method.
\end{abstract}

Keywords: Markovian jump systems; sliding mode control; observer design; event-triggering scheme

\section{Introduction}

With the strong demands for modeling a physical system with high accuracy, for instance, the physical systems may have structural mutation due to changes of power, the shift of parameters and external disturbances, etc., which provoke the need to seek for more suitable mathematical models. Therefore, Markovian jump systems (MJSs) have attracted numerous attention because of its ability to model such kind of physical model with multimodal characteristics or intelligent control system with multi-controller switching [1]. So far, the researches on MJSs have been reported by many in both applications and theoretical aspects, such as the Markov jump models are widely applied in nuclear power systems, manufactory and network communication [2-5], etc. Theoretically, it also witnessed the issue of stochastic stability analysis, stabilization, filter design and so on. For example, in [6], the robust stability and control of uncertain discrete-time linear systems with Markovian jumping parameters was dealt with; in [7], the problems of stochastic stabilization and $H_{\infty}$ control for 2-D MJSs were proposed; and systematic theory on stochastic differential equations with Markovian switching was presented in [8]; the $H_{\infty}$ filtering problem for MJSs was studied in [9,10]; for more details, we may refer to [11-15] and some of their references. In another aspect, due to the existence of nonlinearity, the Takagi-Sugeno (T-S) fuzzy modeling approach has become one of the most popular and effective ways to handle the synthesis of complex nonlinear systems [16], and the investigation on T-S fuzzy model-based MJSs is also rich. In addition, the stabilization of nonlinear singular MJS 
with matched/unmatched uncertainties based on the T-S fuzzy model was studied in [17]; the robust $H_{\infty}$ control involving a class of uncertain stochastic MJSs was investigated in [18], etc. [19-21]. As we all know, the transition rates (TRs) play a big role in the system's performance, but getting exact TR information seems impossible due to all kinds of limits in actual systems, such as high cost, technique limitations and so on. Hence, it is necessary to study MJSs in the presence of deficient TR information. Up to now, some results can be found in dealing with this issue, but not enough. For example, some pioneer works dealt with the issues of stabilization, stochastic stability and quantized filtering for (singular) MJSs with deficiency mode information in [22-25]. However, all the results proposed in literature [22-25] need TR information, no matter partially or fully. So, what if the information of TRs for one mode to another is completely unknown? This is the new challenge we are going to deal with in this paper.

Sliding mode control (SMC) has exclusive advantages in dealing with the nonlinear complex system, such as the good transient performance, fast response speed and strong robustness, which has attracted great attention in the community since its appearance. The researches on SMC of MJSs were also active, for instance, a class of continuous-time MJSs with digital communication constraints via SMC approach was proposed in [26]; the robust $H_{\infty}$ SMC for discrete-time MJSs subject to intermittent observations was researched in [27]; in Ref. [28], the research of asynchronous SMC was investigated based on uncertain MJSs with time-varying delays. In the presence of status components unavailable, observerbased SMC arises, such as an adaptive sliding mode observer was designed for nonlinear MJSs in [29]; the research about sensor fault estimation along with fault-tolerant control for time-delay MJSs through sliding mode observer technique was studied in [30]; in [31], a reduced-order sliding mode observer was designed to realize adaptive control of T-S fuzzy modeled-based MJSs. More works can be found in [32-34] and references therein.

At present, the control systems are realized by distributed components connected through the network, which could improve efficiency and reliability. Traditionally, the timetriggered control is performed periodically that may bring a heavy communication burden. Recently, the event-triggered control strategy has attracted numerous attention for the transmission of data only allowed when it is necessary, which could save communication resources and make full use of congested network channel. Therefore, the approach of event-triggered control is becoming more and more popular, and the results have been reported involving continuous-time and discrete-time systems [35-38], multi-agent systems [39], stochastic systems [40] and neural networks [41], etc. Recently, the eventtriggered based SMC was also touched in the field, such as the event-triggered SMC for stochastic systems on the way of output feedback was studied in [42], etc. However, the problem of observer-based SMC through event-triggered mechanism is seldom touched yet since such an issue is a difficulty due to network-induced constraints. For instance, how to overcome the network-induced delays in constructing desire state observer in order to realize good approximation of original state, and how to design sliding surface to accommodate the sliding motion have partially stimulated our research.

Briefly speaking, this paper will handle the problem of how to design a sliding mode observer for stabilization of nonlinear MJSs based on T-S fuzzy modeling approach. Most of main attention will be focused on designing an event-triggered based time-delay sliding mode observer and a novel sliding mode surface, then establishing feasible easy-checking LMI conditions to ensure $H_{\infty}$ performance in sliding mode dynamics and error dynamics with totally unknown mode transition information. The main contributions of this paper can be concluded as: (1) Compared with traditional observer, the proposed event-triggered time-delay state observer brings the benefits that error is better suppressed and better stabilization property is obtained; (2) a novel sliding surface function is proposed, based on which the observer gain matrices can be computed in the design process rather than given as in [42]; (3) a new method is proposed to give feasible strict LMI conditions for stability of MJSs with totally unknown transition information; and (4) fuzzy SMC law ensures 
finite-time reachability of sliding surface and keeps sliding motion of each sub-models in the presence of uncertain transition information and nonlinearities.

Notions: In this paper, the concept $X>0(X \geq 0)$ denotes $X$ is a positive definite (positive semidefinite) matrix. $I$ and 0 represent an identity matrix and zero matrix, respectively. $\mathbb{E}(\cdot)$ represents the expectation operator about probability measures. $\|\cdot\|$ represents the Euclidean vector norm. $*$ denotes Symmetric elements in a symmetric matrix. $\operatorname{sym}\{P\}$ expresses $P^{T}+P$.

\section{Model Establishing and Problem Statement}

Let us consider the model of single-link robot arm model proposed in [43], where the equation of dynamic is given by

$$
\ddot{\theta}(t)=-\frac{M g L}{J} \sin (\theta(t))-\frac{D(t)}{J} \dot{\theta}(t)+\frac{1}{J} u(t)
$$

the meanings of parameters are defined in Table 1.

Table 1. NOTATIONS.

\begin{tabular}{cc}
\hline Symbol & Meaning \\
\hline$\vartheta(t)$ & angle position of the robot arm \\
$u(t)$ & the input of control \\
$D(t)$ & coefficient of viscous friction \\
$L$ & length of the arm \\
$J$ & moment of inertia \\
$M$ & mass of the pay load \\
$g$ & the acceleration of gravity \\
\hline
\end{tabular}

Letting $x_{1}(t)=\vartheta(t)$ and $x_{2}(t)=\dot{\vartheta}(t)$. According to the method proposed in [44] that under certain angle position, the nonlinearity $\sin \left(x_{1}(t)\right)$ can be replaced by

$$
\sin \left(x_{1}(t)\right)=h_{1}\left(x_{1}(t)\right) x_{1}(t)+\beta h_{2}\left(x_{1}(t)\right) x_{1}(t)
$$

in which $\beta=0.1 / \pi$ is a known parameter and the membership functions satisfy $h_{1}\left(x_{1}(t)\right)+$ $h_{2}\left(x_{1}(t)\right)=1$ with $h_{1}\left(x_{1}(t)\right), h_{2}\left(x_{1}(t)\right) \in[0,1]$. By solving the above equation, the membership functions $h_{1}\left(x_{1}(t)\right)$ and $h_{2}\left(x_{1}(t)\right)$ are obtained as

$$
\begin{aligned}
& h_{1}\left(x_{1}(t)\right)=\left\{\begin{array}{cc}
\frac{\sin \left(x_{1}(t)\right)-\beta x_{1}(t)}{x_{1}(t)(1-\beta)}, & x_{1}(t) \neq 0 \\
1, & x_{1}(t)=0
\end{array}\right. \\
& h_{2}\left(x_{1}(t)\right)=\left\{\begin{array}{cc}
\frac{x_{1}(t)-\sin \left(x_{1}(t)\right)}{x_{1}(t)(1-\beta)}, & x_{1}(t) \neq 0 \\
0, & x_{1}(t)=0
\end{array}\right.
\end{aligned}
$$

Based on the membership function mentioned above, it is easy to see that if $x_{1}(t)$ is about $0 \mathrm{rad}$, then $h_{1}\left(x_{1}(t)\right)=1, h_{2}\left(x_{1}(t)\right)=0$, if $x_{1}(t)$ is about $-\pi$ or $\pi \mathrm{rad}$, then $h_{1}\left(x_{1}(t)\right)=0$ and $h_{2}\left(x_{1}(t)\right)=1$. Hence, the fuzzy model-based state-space description of the robotic system can be rewritten as:

Plant Rule 1: IF $x_{1}(t)$ is "about $0 \mathrm{rad}^{\text {", }}$

THEN

$$
\dot{x}(t)=A_{1} x(t)+B u(t)
$$

where $x(t)=\left[\begin{array}{ll}x_{1}^{T}(t) & x_{2}^{T}(t)\end{array}\right]^{T}$ and

$$
A_{1}=\left[\begin{array}{cc}
0 & 1 \\
-\frac{M g L}{J} & -\frac{D(t)}{J}
\end{array}\right], A_{2}=\left[\begin{array}{cc}
0 & 1 \\
-\frac{\beta M g L}{J} & -\frac{D(t)}{J}
\end{array}\right], B=\left[\begin{array}{c}
0 \\
\frac{1}{J}
\end{array}\right]
$$


Particularly, as discussed in [43], the moment of inertia $J$ may change due to the abrupt change of actual operating, for instance, the switching of the parameters follows the Markovian jumping rules. Therefore, based on the above model, let us consider the following general model:

Plant Rule i: IF $\vartheta_{1}(t)$ is $F_{i 1}$ and $\vartheta_{2}(t)$ is $F_{i 2}$ and $\ldots$ and $\vartheta_{p}(t)$ is $F_{i p}$

THEN

$$
\left\{\begin{array}{l}
\dot{x}(t)=A_{i}\left(r_{t}\right) x(t)+B\left(r_{t}\right) u(t) \\
y(t)=C\left(r_{t}\right) x(t)
\end{array}\right.
$$

where $x(t) \in \mathcal{R}^{n}$ denotes the state vector; $\vartheta_{1}(t), \cdots, \vartheta_{p}(t)$ are premise variables; $F_{i j}$ $(i=1,2, \cdots, r ; j=1,2, \cdots p)$ are fuzzy sets, $u(t) \in \mathcal{R}^{l}$ is the control input; $y(t) \in \mathcal{R}^{q}$ is the controlled output. $A_{i}\left(r_{t}\right)$ and $C\left(r_{t}\right)$ are the system matrices with appropriate dimensions; $B\left(r_{t}\right)$ is the input matrix with full column rank. The process of stochastic jumping $\left\{r_{t}, t \geq 0\right\}$ is a continuous-time homogenous Markovian jumping process. The process of generator with values in a finite set $\mathcal{S}=\{1,2, \cdots, s\}$ given by

$$
\operatorname{Pr}\left\{r_{t+h}=n \mid r_{t}=m\right\}= \begin{cases}\pi_{m n} h+o(h), & m \neq n, \\ 1+\pi_{m m} h+o(h), & m=n\end{cases}
$$

where $h>0$ and $\lim _{h \rightarrow 0} O(h) / h=0, \pi_{m n}(h)>0, m \neq n$, is the transition rate from mode $m$ at time $t$ to mode $n$ at time $t+h$. Meanwhile, $\pi_{m m}=-\sum_{n \neq m} \pi_{m n}<0$ for each $m \in \mathcal{S}$.

Through fuzzy standard blending, the model can be easily derived:

$$
\dot{x}(t)=\sum_{i=1}^{r} h_{i}(\vartheta(t))\left[A_{i}\left(r_{t}\right) x(t)+B\left(r_{t}\right) u(t)\right]
$$

in which $\vartheta(t)=\left[\begin{array}{llll}\vartheta_{1}(t) & \vartheta_{2}(t) & \cdots & \vartheta_{p}(t)\end{array}\right]^{T}, h_{i}(\vartheta(t))$ is the membership function that the formula of which is

$$
h_{i}(\vartheta(t))=\frac{\Pi_{j=1}^{p} \mu_{i j}\left(\vartheta_{j}(t)\right)}{\sum_{i=1}^{r} \Pi_{j=1}^{p} \mu_{i j}\left(\vartheta_{j}(t)\right)}
$$

where $\mu_{i j}\left(\vartheta_{j}(t)\right)$ is the rank of membership of $\vartheta_{j}(t)$ in $\mu_{i j}$. In addition, for all $t>0$, it is satisfied that $h_{i}(\vartheta(t)) \geq 0$ and $\sum_{i=1}^{r} h_{i}(\vartheta(t))=1$. For simplicity, $r(t) \triangleq m$ and $h_{i}(\vartheta)$ is short for $h_{i}(\vartheta(t))$ in the following.

The following Definitions and Lemma are introduced.

Definition 1. ([45]) Given the Lyapunov functional candidate $V\left(x(t), r_{t}, t \geq 0\right)$ with twice differentiable on $x(t)$, then, $\mathcal{L} V\left(x(t), r_{t}\right)$ which is infinitesimal operator is given by

$$
\mathcal{L} V\left(x(t), r_{t}\right)=\lim _{\delta \rightarrow 0} \frac{\mathbb{E}\left\{V\left(x(t+\delta), r_{t+\delta} \mid x(t), r_{t}=m\right)\right\}-V(x(t), m)}{\delta}
$$

Definition 2. ([46]) The unforced stochastic system (2) (i.e., $u(t)=0$ ) is said to be stochastically stable for any initial condition $x\left(t_{0}\right)$ and $t_{t_{0}}$, as long as it satisfies

$$
\lim _{t \rightarrow+\infty} \mathbb{E}\left\{\int_{t_{0}}^{t}\|x(s)\|^{2} d s \mid x\left(t_{0}\right), r_{t_{0}}\right\}<+\infty
$$

Lemma 1. ([47]) Given any real number $\varepsilon$ and any square matrix $R$, for any matrix $F>0$, the matrix inequality $\varepsilon\left(R+R^{T}\right) \leq \varepsilon^{2} F+R F^{-1} R^{T}$ holds.

Here, in this paper, an appropriate observer-based fuzzy SMC strategy for the model (2) on the communication networks with network-induced constraints has been designed to obtain good stochastic stability property with an $H_{\infty}$ performance in sliding mode dynamics and error dynamics. 


\section{Main Results}

Given that the state component may not be accessible in practice, a state observer needs to be designed first. Through digital communication medium, the signal is transmitted in the output channel of observer that may suffer from network-induced delays, where the output measurement $y(t)$ sampled periodically with sampling period $T$. Here, $y(i T)$ $(i=0,1,2, \cdots, \infty)$ is the current measurement and $y\left(i_{k} T\right)\left(i_{k} \in N, k=0,1,2, \cdots, \infty\right.$, $i_{0}=0, k$ is triggering time) is the one latest transmitted, respectively. The judgement condition whether retrieving the transmission is determined by the following conditions:

$$
\left[y\left(\left(i_{k}+j\right) T\right)-y\left(i_{k} T\right)\right]^{T} \Omega_{m}\left[y\left(\left(i_{k}+j\right) T\right)-y\left(i_{k} T\right)\right]>\rho y^{T}\left(i_{k} T\right) \Omega_{m} y\left(i_{k} T\right)
$$

where $\Omega_{m}$ is a positive definite weighting matrix decided by the error tolerance $\rho \in[0,1)$.

The transmission scheme in the next transmission instant $i_{i+1} T$ is determined by

$$
i_{k+1} T=i_{k} T+\min _{j \geq 1}\left\{j T \mid\left[y\left(\left(i_{k}+j\right) T\right)-y\left(i_{k} T\right)\right]^{T} \Omega_{m}\left[y\left(\left(i_{k}+j\right) T\right)-y\left(i_{k} T\right)\right]>\rho y^{T}\left(i_{k} T\right) \Omega_{m} y\left(i_{k} T\right)\right\}
$$

Remark 1. From the above triggered mechanism, only meeting the triggering condition that data packets sampled will be sent on the network; therefore, the network efficiency is improved. In particular, if $\rho=0$, it is seen that the data transmission mechanism is simplified from event triggering to periodic triggering.

\subsection{Network and $\mathrm{ZOH}$}

Due to network transmission speed, network transmission protocol and the load connected in the network, the network-induced delays are considered first, the released transmission delay in kth is marked as $d_{k}$, that is, $d_{k}=t_{k}-i_{k} T$, where $t_{k}$ is the instance that the measurement $y\left(i_{k} T\right)$ arrived $\mathrm{ZOH}$. Let $d_{m}$ be the maximum transmission delay, i.e., $d_{m}=\max \left\{d_{k} \mid k=0,1,2, \cdots, \infty\right\}$. Therefore, the interval of time $\left[t_{k}, t_{k}+1\right)$ can be divided into subintervals as follows:

$$
\left\{\begin{array}{l}
I_{1}=\left[t_{k}, t_{k}+T\right), \\
I_{2}=\left[t_{k}+T, t_{k}+2 T\right), \\
\vdots \\
I_{\tilde{j} k}=\left[t_{k}+\left(\widetilde{j}_{k}-1\right) T, t_{k+1}\right),
\end{array}\right.
$$

where $\widetilde{j_{k}}$ is determined by $\widetilde{j_{k}}=\min \left\{j \mid t_{k}+(j-1) T \geq t_{k+1}, j=1,2, \cdots\right\}$. Then

$$
\left[t_{k}, t_{k_{1}}\right)=\cup_{j=1}^{\widetilde{k_{k}}} I_{j}
$$

Denote

$$
\tau(t)=\left\{\begin{array}{l}
t-i_{k} T, t \in I_{1} \\
t-\left(i_{k}+1\right) T, t \in I_{2} \\
\vdots \\
t-\left(i_{k}+\left(\widetilde{j}_{k}-1\right) T\right), t \in I_{\tilde{j}_{k}}
\end{array}\right.
$$

Obviously,

$$
0 \leq \tau(t)<d_{k}+T \leq d_{m}+T \triangleq \bar{d}_{m}
$$

Now, define

$$
\left\{\begin{array}{l}
0, t \in I_{1}, \\
y\left(i_{k} T\right)-y\left(\left(i_{k}+1\right) T\right), t \in I_{2}, \\
\vdots \\
y\left(i_{k} T\right)-y\left(i_{k}+\left(\tilde{j}_{k}-1\right) T\right), t \in I_{\tilde{j}_{k}}
\end{array}\right.
$$


which combines with (9) leads to

$$
y\left(i_{k} T\right)=e_{k}(t)+y(t-\tau(t))
$$

Given the triggering situation (7), $y\left(\left(i_{k}+j\right) T\right)$ satisfies that

$$
e_{k}^{T}(t) \Omega_{m} e_{k}(t) \leq \rho\left[e_{k}(t)+y(t-\tau(t))\right]^{T} \Omega_{m}\left[e_{k}(t)+y^{T}(t-\tau(t))\right]
$$

In addition, as shown in Figure 1 that the input $\bar{y}(t)$ in state observer satisfies

$$
\bar{y}(t)=y\left(i_{k} T\right), t \in\left[t_{k}, t_{k+1}\right)
$$

that is the received signal equals to the signal released at instants of event-triggering $t_{0}<t_{1}<t_{2}<\cdots<t_{k}<\cdots$. The output signal from $\mathrm{ZOH}$ is piecewise constant but continuous from the right. Thanks to the characteristics of $\mathrm{ZOH}$ causal reconstruction, it is convenient to carry out the design of observer and the analysis of sliding motion.

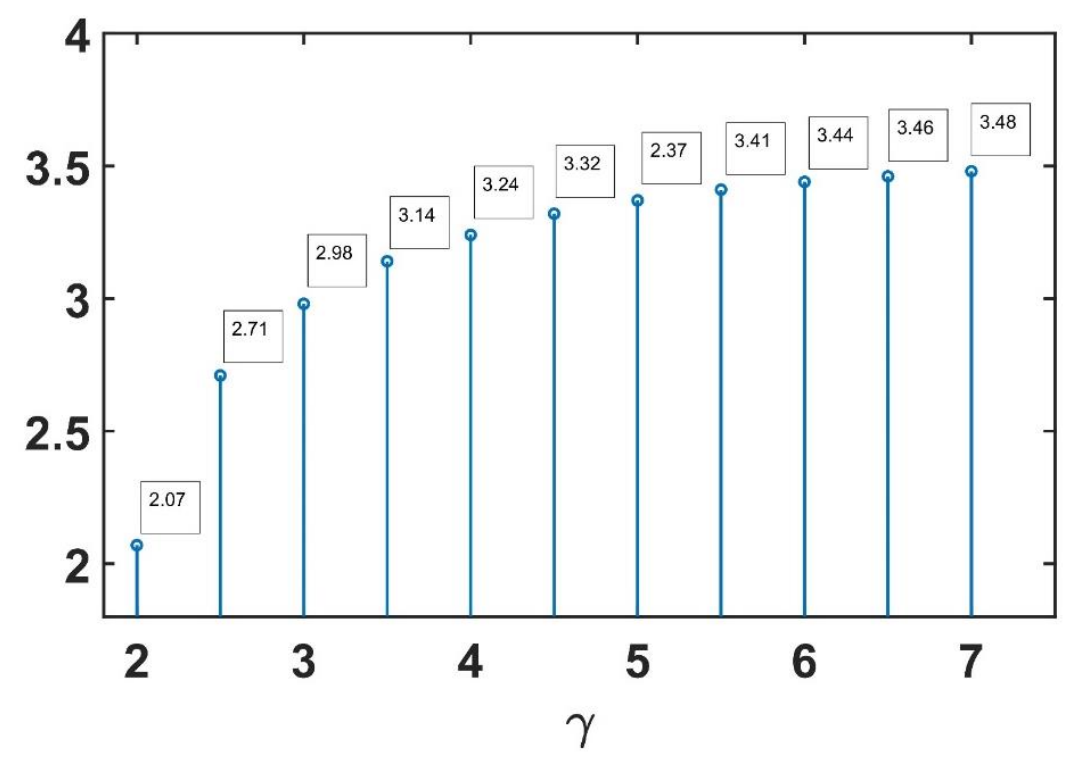

Figure 1. Maximum $a_{3}$ for different $\gamma$.

\subsection{Luenberger State Observer Design}

The premise variable is also unavailable which is considered in the paper. Therefore, according to the measurement $\bar{y}(t)$ for $t \in\left[t_{k}, t_{k+1}\right)(k=0,1,2, \cdots, \infty)$, it is easy to see as follows.

Observer Rule i: IF $\hat{\vartheta}_{1}(t)$ is $M_{i 1}$ and $\hat{\vartheta}_{2}(t)$ is $M_{i 2}$ and $\ldots$ and $\hat{\vartheta}_{n}(t)$ is $M_{i p}$

THEN

$$
\left\{\begin{array}{l}
\dot{\hat{x}}(t)=A_{i, m} \hat{x}(t)+B_{m} u(t)+L_{i, m}(\bar{y}(t)-\hat{y}(t-\tau(t))) \\
\hat{y}(t)=C_{m} \hat{x}(t)
\end{array}\right.
$$

where $\hat{x}(t)$ is the estimation of the state $x(t) . L_{i, m}$ is the observer gain having appropriate dimensions to be determined.

In addition, the dynamics of fuzzy observer (14) after fuzzy blending is inferred as

$$
\dot{\hat{x}}(t)=\sum_{i=1}^{r} h_{i}(\hat{\vartheta})\left[A_{i, m} \hat{x}(t)+B_{m} u(t)+L_{i, m}(\bar{y}(t)-\hat{y}(t-\tau(t)))\right]
$$


It can be seen from (11) and (13) that $\bar{y}(t)=e_{k}(t)+y(t-\tau(t))$. Moreover, the formula of the fuzzy observer is given as follows:

$$
\dot{\hat{x}}(t)=\sum_{i=1}^{r} h_{i}(\hat{\vartheta})\left[A_{i, m} \hat{x}(t)+B_{m} u(t)+L_{i, m}\left(e_{k}(t)+y(t-\tau(t))-\hat{y}(t-\tau(t))\right)\right]
$$

Define $e(t)=x(t)-\hat{x}(t)$ as estimated error. Combining (3) with (16), it is easy to obtain:

$$
\left\{\begin{array}{l}
\dot{e}(t)=\sum_{i=1}^{r} h_{i}(\hat{\vartheta})\left[A_{i, m} e(t)-L_{i, m} C_{m} e(t-\tau(t))-L_{i, m} e_{k}(t)\right]+w(t) \\
y_{e}(t)=C_{m} e(t)
\end{array}\right.
$$

where $w(t)=\sum_{i=1}^{r}\left(h_{i}(\vartheta)-h_{i}(\hat{\vartheta})\right)\left[A_{i, m} x(t)+B_{m} u(t)\right]$ is regarded as the disturbance. Obviously, if $\vartheta(t)=\hat{\vartheta}(t)$, then $w(t)=0$.

Remark 2. Combined with formulas (16) and (17), it is easy to obtain that the addition of time-delay term makes the effect of sliding mode control better and can better suppress the error.

\subsection{Sliding Surface Design}

On the basis of (16), we propose a fuzzy integral sliding surface function:

$$
s(t)=G \hat{x}(t)-\int_{0}^{t} \sum_{i=1}^{r} h_{i}(\hat{\vartheta}) G\left[\left(A_{i, m}+\lambda_{m} B_{m} K_{i, m}\right) \hat{x}(s)+\left(1-\lambda_{m}\right) B_{m} u(s)\right] d s
$$

where $G \in \mathcal{R}^{m \times n}$ making $G B_{m}$ nonsingular is chosen, $\lambda_{m}$ making $I-\frac{1}{\lambda_{m}} B_{m}\left(G B_{m}\right)^{-1} G$ nonsingular is given. $K_{i, m}$ making $A_{i, m}+B_{m} K_{i, m}$ is Hurwitz is selected.

In view of the systems (16) and (18), as shown as follows

$$
\begin{aligned}
\dot{s}(t) & =-\sum_{i=1}^{r} h_{i}(\hat{\vartheta}) \lambda_{m} G B_{m} K_{i, m} \hat{x}(t)+\lambda_{m} G B_{m} u(t) \\
& +G L_{i, m}\left(e_{k}(t)+C_{m} e(t-\tau(t))\right)
\end{aligned}
$$

After arriving the sliding surface $s(t)=0$, i.e., $\dot{s}(t)=0$, the equivalent control variable can be obtained as follows:

$$
u_{e q}(t)=\sum_{i=1}^{r} h_{i}(\hat{\vartheta})\left[K_{i, m} \hat{x}(t)-\frac{1}{\lambda_{m}}\left(G B_{m}\right)^{-1} G L_{i, m}\left(e_{k}(t)+C_{m} e(t-\tau(t))\right)\right]
$$

Then, by substituting (20) into (16), it is easy to obtain:

$$
\dot{\hat{x}}(t)=\sum_{i=1}^{r} h_{i}(\hat{\vartheta})\left[\bar{A}_{i, m} \hat{x}(t)+\mathcal{I}_{m} L_{i, m}\left(e_{k}(t)+C_{m} e(t-\tau(t))\right)\right]
$$

where $\bar{A}_{i, m}=A_{i, m}+B_{m} K_{i, m}$ and $\mathcal{I}_{m}=I-\frac{1}{\lambda_{m}} B_{m}\left(G B_{m}\right)^{-1} G$.

Remark 3. By selecting appropriate $\lambda_{m}$ such that $\mathcal{I}_{m}$ is invertible. The benefit is that we can obtain the observer gain matrices by solving the optimal problem of the following conditions rather than by given in the design process.

Here, designing a sliding mode controller based on observer for the system (2) is the purpose of this paper and the controller can meet two conditions as follows: 
1. It is stochastically stable for system (17) with $w(t)=0$ and the sliding mode dynamics (21).

2. The measurement of $H_{\infty}$ performance with the condition of zero-initial will be satisfied as follows:

$$
J=\mathbb{E} \int_{0}^{+\infty}\left[y_{e}^{T}(s) y_{e}(s)-\gamma^{2} w^{T}(s) w(s)\right] d s<0
$$

where $\gamma$ is a positive constant.

Remark 4. By selecting this integral sliding mode functional, the linear LMI is obtained for the obtained sliding mode stochastic stability analysis, so that the gain matrix of the observer can be obtained instead of artificial design, which reduces the conservatism.

\subsection{Stochastic Stability and $H_{\infty}$ Performance Analysis}

Remark 5. Given positive scalars $d_{m}, \gamma, a_{1 m}, a_{2 m}$ and $\rho \in[0,1)$, the error dynamics (17) and the sliding mode dynamics (21) are stochastically stable with an $H_{\infty}$ attenuation level $\gamma$, if matrices $P_{m}>0, Q_{1}>0, Q_{2}>0, R_{1}>0, R_{2}>0, \Omega_{m}>0$, free weighting matrices $S_{k m}(k=1,2,3)$ and $Y_{i m}$ with appropriate dimensions exist, the following condition is satisfied for each $m \in \mathcal{S}$ :

$$
\left[\begin{array}{cccc}
\mathcal{A}_{i, m}^{11} & \mathcal{A}_{i, m}^{12} & \mathcal{A}_{i, m}^{13} & 0 \\
* & \mathcal{A}_{i, m}^{22} & 0 & \mathcal{A}_{i, m}^{24} \\
* & * & \mathcal{A}_{i, m}^{33} & 0 \\
* & * & * & \mathcal{A}_{i, m}^{44}
\end{array}\right]<0
$$

where

$$
\begin{aligned}
& \mathcal{A}_{i, m}^{11}=\left[\begin{array}{cccc}
\overline{\mathcal{A}}_{i, m}^{11} & -S_{1 m} & 0 & a_{1 m} \bar{A}_{i, m} I_{m}^{-1} P_{m}^{T} \\
* & \mathcal{D}_{m} & -S_{2 m} & 0 \\
* & * & -Q_{1} & 0 \\
* & * & * & d_{m} R_{1}-\operatorname{sym}\left\{a_{m} P_{m} I_{m}\right\}
\end{array}\right] \\
& \mathcal{A}_{i, m}^{22}=\left[\begin{array}{cccccc}
\overline{\mathcal{A}}_{i, m}^{22} & \mathcal{B}_{i, m}^{11} & 0 & a_{m} \overline{\mathcal{A}}_{i, m}^{T} P_{m}^{T} & \mathcal{B}_{i, m}^{12} & P_{m} \\
* & \mathcal{B}_{i, m}^{13} & -S_{4 m} & C_{m}^{T} Y_{i, m}^{T} & 0 & 0 \\
* & * & -Q_{2} & 0 & 0 & 0 \\
* & * & * & \mathcal{B}_{i, m}^{14} & \mathcal{B}_{i, m}^{15} & a_{2 m} P_{m} \\
* & * & * & * & \mathcal{B}_{i, m}^{16} & 0 \\
* & * & * & * & * & -\gamma^{2} I
\end{array}\right] \\
& \mathcal{A}_{12}=\left[\begin{array}{cccccc}
0 & 0 & 0 & 0 & 0 & 0 \\
0 & C_{m}^{T} \Omega_{m} C_{m} & 0 & C_{m} \Omega_{m} & 0 & 0 \\
0 & 0 & 0 & 0 & 0 & 0 \\
0 & a_{1 m} Y_{i, m} C_{m} & 0 & 0 & a_{1 m} Y_{i, m} & 0
\end{array}\right] \\
& \mathcal{A}_{13}=\left[\begin{array}{ccc}
S_{1 m} & 0 & P_{m} I_{m} \\
0 & S_{2 m} & 0 \\
0 & 0 & 0 \\
0 & 0 & 0
\end{array}\right] \\
& \mathcal{A}_{24}=\left[\begin{array}{ccc}
S_{3 m} & 0 & 0 \\
0 & S_{4 m} & C_{m}^{T} Y_{i, m}^{T} \\
0 & 0 & 0 \\
0 & 0 & 0 \\
0 & 0 & 0 \\
0 & 0 & 0
\end{array}\right]
\end{aligned}
$$




$$
\begin{gathered}
\mathcal{A}_{i, m}^{33}=\operatorname{diag}\left\{-R_{1} / \bar{d}_{m},-R_{1} / \bar{d}_{m},-P_{m} / 2\right\} \\
\mathcal{A}_{i, m}^{44}=\operatorname{diag}\left\{-R_{2} / \bar{d}_{m},-R_{2} / \bar{d}_{m},-P_{m}\right\} \\
\overline{\mathcal{A}}_{i, m}^{11}=\operatorname{sym}\left\{P_{m} \bar{A}_{i, m}+S_{1, m}\right\}+Q_{1}+\sum_{n=1}^{s} \pi_{m n} P_{n} \\
\mathcal{A}_{i, m}^{22}=\operatorname{sym}\left\{P_{m} \bar{A}_{i, m}+S_{3, m}\right\}+Q_{2}+C_{m}^{T} C_{m}+\sum_{n=1}^{s} \pi_{m n} P_{n} \\
\mathcal{D}_{m}=\operatorname{sym}\left\{S_{2 m}\right\}+C_{m}^{T} \Omega_{m} C_{m} \mathcal{B}_{i, m}^{11}=Y_{i, m} C_{m}-S_{3 m} \\
\mathcal{B}_{i, m}^{12}=C_{m}^{T} \Omega_{m}-Y_{i, m} \\
\mathcal{B}_{i, m}^{13}=S_{4 m}+S_{4 m}^{T}+C_{m}^{T} \Omega_{m} C_{m} \\
\mathcal{B}_{i, m}^{14}=d_{m} R_{2}-a_{2 m} P_{m} \\
\mathcal{B}_{i, m}^{15}=-a_{2 m} Y_{i, m} \mathcal{B}_{i, m}^{16}=(\rho-1) \Omega_{m}
\end{gathered}
$$

The observer gain matrices are computed by $L_{i, m}=P_{m}^{-1} Y_{i, m}$.

Proof. First, the overall closed-loop system is stochastically stable with $w(t)=0$ will be proved. Therefore, choose the following stochastic Lyapunov-Krasovskii functional:

$$
V\left(\hat{x}(t), e(t), r_{t}\right)=V\left(\hat{x}(t), r_{t}\right)+V\left(e(t), r_{t}\right)
$$

where

$$
\begin{gathered}
V\left(\hat{x}(t), r_{t}\right)=\hat{x}^{T}(t) P\left(r_{t}\right) \hat{x}(t)+\int_{t-d_{m}}^{t} \hat{x}^{T}(s) Q_{1} \hat{x}(s) d s+\int_{-d_{m}}^{0} \int_{t-\theta}^{t} \dot{\hat{x}}^{T}(s) R_{1} \dot{\hat{x}}(s) d s d \theta \\
V\left(e(t), r_{t}\right)=e^{T}(t) P\left(r_{t}\right) e(t)+\int_{t-d_{m}}^{t} e^{T}(s) Q_{2} e(s) d s+\int_{-d_{m}}^{0} \int_{t-\theta}^{t} \dot{e}^{T}(s) R_{1} \dot{e}(s) d s d \theta
\end{gathered}
$$

By employing Definition 1, it obtains

$$
\begin{aligned}
\mathcal{L} V(\hat{x}(t), m)= & 2 \hat{x}^{T}(t) P_{m} \sum_{i=1}^{r} h_{i}(\hat{\vartheta})\left[\bar{A}_{i, m} \hat{x}(t)+\mathcal{I}_{m} L_{i, m}\left(e_{k}(t)+C_{m} e(t-\tau(t))\right)\right] \\
& +\hat{x}^{T}(t) \sum_{n=1}^{s} \pi_{m n} P_{m} \hat{x}(t)+\hat{x}^{T}(t) Q_{1} \hat{x}(t)+d_{m} \dot{\hat{x}}^{T}(t) R_{1} \dot{\hat{x}}(t) \\
& -\hat{x}^{T}\left(t-d_{m}\right) Q_{1} \hat{x}\left(t-d_{m}\right)-\int_{t-d_{m}}^{t} \dot{\hat{x}}^{T}(s) R_{1} \dot{\hat{x}}(s) d s
\end{aligned}
$$

and

$$
\begin{aligned}
\mathcal{L} V(e(t), m) & =2 e^{T}(t) P_{m} \sum_{i=1}^{r} h_{i}(\hat{\vartheta})\left[A_{i, m} e(t)-L_{i, m} e_{k}(t)-L_{i, k} C e(t-\tau(t))\right]+e^{T}(t) \sum_{n=1}^{s} \pi_{m n} P_{m} e(t) \\
& +e^{T}(t) Q_{2} e(t)-e^{T}\left(t-d_{m}\right) Q_{2} e\left(t-d_{m}\right)+d_{m} \dot{e}^{T}(t) R_{2} \dot{e}(t)-\int_{t-d_{m}}^{t} \dot{e}^{T}(s) R_{2} \dot{e}(s) d s
\end{aligned}
$$

in which

$$
\begin{aligned}
2 \hat{x}^{T}(t) P_{m} \mathcal{I}_{m} L_{i, m} e_{k}(t) \leq \hat{x}^{T}(t) P_{m} \mathcal{I}_{m} P_{m}^{-1} \mathcal{I}_{m}^{T} P_{m} \hat{x}^{T}(t)+e_{k}^{T}(t) L_{i, m}^{T} P L_{i, m} e_{k}(t) \\
\begin{aligned}
2 \hat{x}^{T}(t) P_{m} \mathcal{I}_{m} L_{i, m} C_{m} e(t-\tau(t)) & \leq \hat{x}^{T}(t) P_{m} \mathcal{I}_{m} P_{m}^{-1} \mathcal{I}_{m}^{T} P_{m} \hat{x}^{T}(t) \\
& +e^{T}(t-\tau(t)) C_{m}^{T} L_{i, m}^{T} P_{m} L_{i, m} C_{m} e(t-\tau(t))
\end{aligned}
\end{aligned}
$$


It is obvious from the Leibniz-Newton formula that

$$
\begin{gathered}
2 \hat{x}^{T}(t) S_{1 m}\left[\hat{x}(t)-\hat{x}(t-\tau(t))-\int_{t-\tau(t)}^{t} \dot{\hat{x}}(s) d s\right]=0 \\
2 \hat{x}^{T}(t-\tau(t)) S_{2 m}\left[\hat{x}(t-\tau(t))-\hat{x}\left(t-d_{m}\right)-\int_{t-d_{m}}^{t-\tau(t)} \dot{\hat{x}}(s) d s\right]=0
\end{gathered}
$$

where $S_{1 m}, S_{2 m}$ are free weighting matrices. Moreover,

$$
\begin{array}{r}
-2 \hat{x}^{T}(t) S_{1 m} \int_{t-\tau(t)}^{t} \dot{\hat{x}}(s) d s \leq \int_{t-\tau(t)}^{t} \dot{\hat{x}}^{T}(s) R_{1} \dot{\hat{x}}(s) d s+\bar{d}_{m} \hat{x}^{T}(t) S_{1 m} R_{1}^{-1} S_{1 m}^{T} \hat{x}(t) \\
-2 \hat{x}^{T}(t-\tau(t)) S_{2 m} \int_{t-\tau(t)}^{t-d_{m}} \dot{\hat{x}}(s) d s \\
\leq \int_{t-d_{m}}^{t-\tau(t)} \dot{\hat{x}}^{T}(s) R_{1} \dot{\hat{x}}(s) d s \\
+\bar{d}_{m} \hat{x}^{T}(t-\tau(t)) S_{2 m} R_{1}^{-1} S_{2 m}^{T} \hat{x}(t-\tau(t))
\end{array}
$$

Similarly,

$$
\begin{gathered}
2 e^{T}(t) S_{3 m}\left[e(t)-e(t-\tau(t))-\int_{t-\tau(t)}^{t} \dot{e}(s) d s\right]=0 \\
2 e^{T}(t-\tau(t)) S_{4 m}\left[e(t-\tau(t))-e\left(t-d_{m}\right)-\int_{t-d_{m}}^{t-\tau(t)} \dot{e}(s) d s\right]=0
\end{gathered}
$$

where $S_{3 m}, S_{4 m}$ are free weighting matrices. Then

$$
\begin{array}{r}
-2 e^{T}(t) S_{3 m} \int_{t-\tau(t)}^{t} \dot{e}(s) d s \leq \int_{t-\tau(t)}^{t} \dot{e}^{T}(s) R \dot{e}(s) d s+\bar{d}_{m} e^{T}(t) S_{3 m} R_{2}^{-1} S_{3 m}^{T} e(t) \\
-2 e^{T}(t-\tau(t)) S_{4 m} \int_{t-\tau(t)}^{t-d_{m}} \dot{e}(s) d s \leq \int_{t-d_{m}}^{t-\tau(t)} \dot{e}^{T}(s) R_{2} \dot{e}(s) d s \\
+\bar{d}_{m} e^{T}(t-\tau(t)) S_{4 m} R_{2}^{-1} S_{4 m}^{T} e(t-\tau(t))
\end{array}
$$

On the other hand, it holds that

$$
\dot{\hat{x}}(t) \sum_{i=1}^{r} h_{i}(\hat{\vartheta}) \alpha_{1 m} P_{m} I_{m}^{-1}\left[\bar{A}_{i, m} \hat{x}(t)+I_{m} L_{i, m}\left(e_{y}(t)+C_{m} e(t-\tau(t))-\dot{\hat{x}}(t)\right)\right]=0
$$

Then

$$
\dot{e}(t) \sum_{i=1}^{r} h_{i}(\hat{\vartheta}) \alpha_{2 m} P_{m}\left[A_{i, m} e(t)-L_{i, m} C_{m} e(t-\tau(t))-L_{i, m} e_{y}(t)-\dot{e}(t)\right]=0
$$

where $\alpha_{1 m}$ and $\alpha_{2 m}$ are chosen parameters. In summary, we have

$$
\mathcal{L} V\left(\hat{x}(t), e(t), r_{t}\right) \leq \sum_{i=1}^{r} h_{i}(\hat{\vartheta}) \eta^{T}(t) \bar{\Phi}_{i, m} \eta(t)
$$

in which

$$
\begin{aligned}
& \eta^{T}(t)=\left[\begin{array}{llll}
x^{T}(t) & x^{T}(t-\tau(t)) & x^{T}\left(t-d_{m}\right) & \dot{x}^{T}(t)
\end{array}\right. \\
& \left.\begin{array}{lllll}
e^{T}(t) & e^{T}(t-\tau(t)) & e^{T}\left(t-d_{m}\right) & \dot{e}^{T}(t) & e_{k}^{T}(t)
\end{array}\right]
\end{aligned}
$$

$$
\Phi_{i, m}=\left[\begin{array}{cc}
\Phi_{i, m}^{1} & \Phi_{i, m}^{2} \\
* & \Phi_{i, m}^{3}
\end{array}\right]
$$


with

$$
\begin{gathered}
\Phi_{i, m}^{1}=\left[\begin{array}{ccccc}
\Phi_{i, m}^{11} & -S_{1 m} & 0 & a_{1 m} A_{i, m} I_{k}^{-1} P_{m}^{T} \\
* & \Phi_{i, m}^{12} & -S_{2 m} & 0 \\
* & * & -Q_{1} & 0 \\
* & * & * & & \Phi_{i, m}^{13}
\end{array}\right] \\
\Phi_{i, m}^{2}=\left[\begin{array}{ccccc}
0 & 0 & 0 & 0 & 0 \\
0 & C_{m}^{T} \Omega_{m} C_{m} & 0 & 0 & C_{m}^{T} \Omega \\
0 & 0 & 0 & 0 & 0 \\
0 & a_{1 m} P_{m} L_{i, m} C_{m} & 0 & 0 & a_{1 m} P_{m} L_{i, m}
\end{array}\right] \\
\Phi_{i, m}^{3}=\left[\begin{array}{cccccc}
\Phi_{i, m}^{31} & \Phi_{i, m}^{32} & 0 & a_{2 m} \bar{A}_{i, m}^{T} P_{m}^{T} & \Phi_{i, m}^{33} \\
* & \Phi_{i, m}^{34} & -S_{4 m} & C_{m}^{T} L_{i, m}^{T} P_{m}^{T} & 0 \\
* & * & -Q_{2} & 0 & 0 \\
* & * & * & \Phi_{i, m}^{35} & \Phi_{i, m}^{36} \\
* & * & * & * & (\rho-1) \Omega_{m}
\end{array}\right]
\end{gathered}
$$

in which

$$
\begin{gathered}
\Phi_{i, m}^{11}=\operatorname{sym}\left\{P_{m} \bar{A}_{i, m}+S_{1 m}\right\}+Q_{1}+\sum_{n=1}^{S} \pi_{m n} P_{n}+\bar{d}_{m} S_{1 m} R_{1}^{-1} S_{1 m}^{T}+2 P_{m} I_{m} P_{m}^{-1} I_{m} P_{m} \\
\Phi_{i, m}^{12}=S_{2 m}+S_{2 m}^{T}+C_{m}^{T} \Omega_{m} C_{m}+\bar{d}_{m} S_{2 m} R_{1}^{-1} S_{2 m}^{T} \\
\Phi_{i, m}^{13}=d_{m} R_{1}-\operatorname{sym}\left\{a_{m} P_{m} I_{m}^{-1}\right\} \\
\Phi_{i, m}^{31}=\operatorname{sym}\left\{P_{m} \bar{A}_{i, m}+S_{3 m}\right\}+Q_{3}+\sum_{n=1}^{s} \pi_{m n} P_{n}+\bar{d}_{m} S_{3 m} R_{2}^{-1} S_{3 m}^{T} \\
\Phi_{i, m}^{32}=P_{m} L_{i, m} C_{m}-S_{3 m} \\
\Phi_{i, m}^{33}=C_{m}^{T} \Omega_{m}-P_{m} L_{i, m} \\
\Phi_{i, m}^{34}=S_{4 m}+S_{4 m}^{T}+C_{m}^{T} \Omega_{m} C_{m}+\bar{d}_{m} S_{4 m} R_{2}^{-1} S_{4 m}^{T}+C_{m}^{T} L_{i, m}^{T} P_{m} L_{i, m} C_{m} \\
\Phi_{i, m}^{35}=d_{m} R_{2}-a_{2 m} P_{m} \\
\Phi_{i, m}^{35}=-a_{2 m} P_{m} L_{i, m}
\end{gathered}
$$

Letting $P_{m} L_{i, m}=Y_{i, m}$ and $\bar{\Phi}_{i, m}<0$ can be known from (22) by Schur complement. So, if a scaler $\mu \triangleq \lambda_{\min }\left\{-\bar{\Phi}_{i, m}\right\}>0$ is denoted, we will know

$$
\mathcal{L} V\left(\hat{x}(t), e(t), r_{t}\right) \leq-\mu\|\hat{x}(t)\|^{2}
$$

Hence, after using Dynkin's formula, some conclusions can be drawn, that is, for $t>0$

$$
\mathbb{E} V\left(\hat{x}(t), e(t), r_{t}\right)-\mathbb{E} V\left(\hat{x}(0), e(0), r_{0}\right) \leq-\mu \mathbb{E} \int_{0}^{t}\|\hat{x}(t)\|^{2} d s
$$

which produces

$$
\mathbb{E} \int_{0}^{t}\|\hat{x}(s)\|^{2} d s \leq \mu^{-1} \mathbb{E} V\left(\hat{x}(0), e(0), r_{0}\right)
$$

Then, according to Definition 1 , it is stochastically stable when $w(t)=0$ for the sliding mode dynamics (21), and it also can be proved for error system (17) in the same way. 
Next, the $H_{\infty}$ performance of overall closed-loop system will be considered. $\mathbb{E} V(t)=\mathbb{E} \int_{0}^{+\infty} \mathcal{L} V(s) d s \geq 0$ in the condition of zero-initial. Therefore,

$$
\begin{aligned}
J & =\mathbb{E} \int_{0}^{+\infty}\left[y_{e}^{T}(s) y_{e}(s)-\gamma^{2} w^{T}(s) w(s)\right] d s \\
& \leq \mathbb{E} \int_{0}^{+\infty}\left[y_{e}^{T}(s) y_{e}(s)-\gamma^{2} w^{T}(s) w(s)+\mathcal{L} V(s)\right] d s \\
& =\mathbb{E} \int_{0}^{+\infty} \sum_{i=1}^{r} h_{i}(\hat{x}(s)) \zeta^{T}(s) \hat{\Phi}_{i, m} \zeta(s) d s
\end{aligned}
$$

in which $\zeta^{T}(t)=\left[\begin{array}{ll}\eta^{T}(t) & w^{T}(t)\end{array}\right]^{T}$, and

$$
\hat{\Phi}_{i, m}=\breve{\Phi}_{i, m}+\operatorname{diag}\left\{\sum_{n=1}^{s} \pi_{m n} P_{n}, 0, \sum_{n=1}^{s} \pi_{m n} P_{n}, 0\right\}
$$

with

$$
\breve{\Phi}_{i, m}=\left[\begin{array}{cc}
\Phi_{i, m}+\operatorname{diag}\left\{0, C_{m}^{T} C_{m}\right\} & {\left[\begin{array}{c}
0 \\
P_{m}
\end{array}\right]} \\
* & -\gamma^{2} I
\end{array}\right]
$$

By utilizing Schur's complement and the inequality (24), obviously, $\hat{\Phi}_{i, m}<0$ means $J<0$. Therefore, it is stochastically stable for the sliding mode dynamics (21) with error dynamics (17) with an $H_{\infty}$ disturbance attenuation level $\gamma$.

Remark 6. Due to various environmental constraints, the TRs information is often not obtained as expected in practice. Therefore, TRS may encounter three situations, where $\pi_{m n}$ is completely known, partially known and completely unknown.

For example, the TR matrix may be presented as follows:

$$
\left[\begin{array}{cccc}
\pi_{11} & ? & \cdots & \hat{\pi}_{1 s}+\Delta \hat{\pi}_{1 s} \\
\pi_{21} & \hat{\pi}_{22}+\hat{\pi}_{22} & \cdots & ? \\
\vdots & \vdots & \ddots & \vdots \\
\pi_{s 1} & \hat{\pi}_{s 2}+\Delta \hat{\pi}_{s 2} & \cdots & ?
\end{array}\right]
$$

in which $\pi_{m n}, \hat{\pi}_{m n}$ and $\Delta \hat{\pi}_{m n} \in\left[-\delta_{m n}, \delta_{m n}\right]$ subject to $0<\delta_{m n}<\left|\pi_{m n}, \hat{\pi}_{m n}\right|$, denote the known values, the estimated values and estimated error of uncertain TRs, respectively. Then "?" denotes TRs that unknown completely. Hereinafter, in order to calculate uniformity, these known TRs are represented by $\pi_{m n} \triangleq \hat{\pi}_{m n}+\Delta \hat{\pi}_{m n}$, where $\Delta \hat{\pi}_{m n}=0$, so, the TR matrix can be described further by

$$
\left[\begin{array}{cccc}
\hat{\pi}_{11}+\Delta \hat{\pi}_{11} & ? & \cdots & \hat{\pi}_{1 s}+\Delta \hat{\pi}_{1 s} \\
\hat{\pi}_{21}+\Delta \hat{\pi}_{21} & \hat{\pi}_{22}+\Delta \hat{\pi}_{22} & \cdots & ? \\
\vdots & \vdots & \ddots & \vdots \\
\hat{\pi}_{s 1}+\Delta \hat{\pi}_{s 1} & \hat{\pi}_{s 2}+\Delta \hat{\pi}_{s 2} & \cdots & ?
\end{array}\right]
$$

where $\Delta \hat{\pi}_{m n} \in\left[-\delta_{m n}, \delta_{m n}\right]$ with $\delta_{m n} \geq 0$. According to the above transformation, the following sets are defined:

$$
I_{m, k}=I_{m, k n} \cup I_{m, u k n},
$$

where

$$
\begin{aligned}
& I_{m, k n} \triangleq\left\{n: \hat{\pi}_{m n} \text { is known for } n \in \mathcal{S}\right\} \\
& I_{m, u k n} \triangleq\left\{n: \hat{\pi}_{m n} \text { is not known for } n \in \mathcal{S}\right\}
\end{aligned}
$$


Based on the above sets, the following situations are considered:

1. $m \in I_{m, k n}$ and $\hat{\pi}_{m n}$ for $\forall n \in I_{m, k n}$ are known, that is $I_{m, k n}$ are known, that is $I_{m, k n}=\mathcal{S}$;

2. $m \in I_{m, k n}$ and $\hat{\pi}_{m n}$ for $\forall n \in I_{m, k n}$ are partially known, that is $I_{m, k n} \neq \mathcal{S}$ while $I_{m, k n}$ is also not empty;

3. $m \in I_{m, u k n}$ and $\hat{\pi}_{m n}$ for $\forall n \in I_{m, k n}$ are partially known, that is $I_{m, k n} \neq \mathcal{S}$ while $I_{m, k n}$ is also not empty;

4. $m \in I_{m, u k n}$ and $\hat{\pi}_{m n}$ for $\forall n \in I_{m, k n}$ are all unknown, that is $I_{m, k n}=\phi$.

It is known that the above cases 1-3 have been investigated in other works, while the cased 4 was neglected, where is the main difficulty lies. Therefore, in this paper, the following method brought from [48] is introduced.

For the case 4, i.e., $m \in I_{m, u k n}$ and $I_{m, k n}=\varnothing$, there exists $n \in I_{n, k n}$ with $I_{n, k n}$ is not empty for $n \neq m$. In this situation, define

$$
\pi_{m m}=a_{m} \pi_{n n}
$$

where $a_{m}$ is the estimated parameter that will be determined.

For example, the three modes TR matrix could be expressed by:

$$
\left[\begin{array}{ccc}
\hat{\pi}_{11}+\Delta \hat{\pi}_{11} & \hat{\pi}_{12}+\Delta \hat{\pi}_{12} & \hat{\pi}_{13}+\Delta \hat{\pi}_{13} \\
\hat{\pi}_{21}+\Delta \hat{\pi}_{21} & \hat{\pi}_{22}+\Delta \hat{\pi}_{22} & \hat{\pi}_{23}+\Delta \hat{\pi}_{23} \\
? & ? & ?
\end{array}\right]
$$

It is seen from the above matrix that $I_{3, k n}$ is empty, while the unknown TR $\pi_{33}$ can be estimated by $\pi_{11}$ or $\pi_{22}$. Based on TRs matrix mentioned above, the theorem will be obtained as follows.

Theorem 2. Given positive scalars $d_{m}, \gamma, a_{1 m}, a_{2 m}$ and $\rho \in[0,1)$. The sliding mode dynamics (21) with the error dynamics (17) is stochastically stable with an $H_{\infty}$ attenuation level $\gamma$, if matrices $P_{m}>0, Q_{1}>0, Q_{2}>0, R_{1}>0, R_{2}>0, U_{m m}>0, W_{m m}>0, \Omega_{m}>0$, free weighting matrices $S_{k m}(k=1,2,3)$ and $Y_{i m}$ with appropriate dimensions exist, the following conditions are satisfied for each $m \in \mathcal{S}$ If $m \in I_{m, k n}$ and $I_{m, k n}=\mathcal{S}$, then

$$
\left[\begin{array}{cccccc}
\Gamma_{i, m}^{11} & \mathcal{A}_{i, m}^{12} & \mathcal{A}_{i, m}^{13} & 0 & \mathcal{A}_{i, m}^{15} & 0 \\
* & \Theta_{i, m}^{11} & 0 & \mathcal{A}_{i, m}^{24} & 0 & \mathcal{A}_{i, m}^{26} \\
* & * & \mathcal{A}_{i, m}^{33} & 0 & 0 & 0 \\
* & * & * & \mathcal{A}_{i, m}^{44} & 0 & 0 \\
* & * & * & * & \mathcal{A}_{i, m}^{55} & 0 \\
* & * & * & * & * & \mathcal{A}_{i, m}^{66}
\end{array}\right]<0,
$$

If $m \in I_{m, u k n}$, there exists $n \neq m$ such that $n \in I_{n, k n}$, for $\forall l \in I_{m, u k n}$,

$$
\left[\begin{array}{cccccc}
\Gamma_{i, m}^{21} & \mathcal{A}_{i, m}^{12} & \mathcal{A}_{i, m}^{13} & 0 & \mathcal{C}_{i, m}^{15} & 0 \\
* & \Theta_{i, m}^{21} & 0 & \mathcal{A}_{i, m}^{24} & 0 & \mathcal{C}_{i, m}^{26} \\
* & * & \mathcal{A}_{i, m}^{33} & 0 & 0 & 0 \\
* & * & * & \mathcal{A}_{i, m}^{44} & 0 & 0 \\
* & * & * & * & -\frac{1}{a_{m}} W_{m m} & 0 \\
* & * & * & * & * & -\frac{1}{a_{m}} W_{m m}
\end{array}\right]<0
$$

where $\Gamma_{i, m}^{k 1}$ is defined as:

$$
\left[\begin{array}{cccc}
\bar{\Gamma}_{i m}^{k 1} & -\mathcal{S}_{1 m} & 0 & a_{1 m} \bar{A}_{i, m} I_{m}^{-1} P_{m}^{T} \\
* & \mathcal{D}_{m} & -\mathcal{S}_{2 m} & 0 \\
* & * & -Q_{1} & 0 \\
* & * & * & d_{m} R_{1}-\operatorname{sym}\left\{a_{m} P_{m} I_{m}\right\}
\end{array}\right]
$$


and, $\Theta_{i, m}^{k 1}$ is defined as:

$$
\left[\begin{array}{cccccc}
\bar{\Theta}_{i, m}^{k 1} & \mathcal{B}_{i, m}^{11} & 0 & a_{m} \bar{A}_{i, m}^{T} P_{m}^{T} & \mathcal{B}_{i, m}^{12} & P_{m} \\
* & \mathcal{B}_{i, m}^{13} & -S_{4 m} & C_{m}^{T} Y_{i, m}^{T} & 0 & 0 \\
* & * & -Q_{2} & 0 & 0 & 0 \\
* & * & * & \mathcal{B}_{i, m}^{14} & \mathcal{B}_{i, m}^{15} & a_{2 m} P_{m} \\
* & * & * & * & \mathcal{B}_{i, m}^{16} & 0 \\
* & * & * & * & * & -\gamma^{2} I
\end{array}\right]
$$

with

$$
\begin{aligned}
& \mathcal{A}_{15}=\mathcal{A}_{26}=\left[\begin{array}{cccccc}
P_{1}-P_{m} & \cdots & P_{m-1}-P_{m} & P_{m+1}-P_{m} & \cdots & P_{s}-P_{m} \\
0 & \cdots & 0 & 0 & \cdots & 0
\end{array}\right] \\
& \bar{\Gamma}_{i, m}^{11}=\operatorname{sym}\left\{P_{m} \bar{A}_{i, m}+S_{1 m}\right\}+Q_{1}+\sum_{n=1}^{s} \pi_{m n} P_{n}+\sum_{n \neq m} \frac{\delta_{m n}^{2}}{4} U_{m n} \\
& \bar{\Gamma}_{i, m}^{21}=\operatorname{sym}\left\{P_{m} \bar{A}_{i, m}+S_{1 m}\right\}+Q_{1}+\frac{\delta_{n n}^{2}}{4} a_{m} W_{m m}+a_{m} \hat{\pi}_{n n}\left(P_{m}-P_{l}\right) \\
& \bar{\Theta}_{i, m}^{11}=\operatorname{sym}\left\{P_{m} \bar{A}_{i, m}+S_{3 m}\right\}+Q_{2}+C_{m}^{T} C_{m}+\sum_{n=1}^{s} \pi_{m n} P_{n}+\sum_{n \neq m} \frac{\delta_{m n}^{2}}{4} U_{m n} \\
& \bar{\Theta}_{i, m}^{21}=\operatorname{sym}\left\{P_{m} \bar{A}_{i, m}+S_{3 m}\right\}+Q_{2}+C_{m}^{T} C_{m}+\frac{\delta_{n n}^{2}}{4} a_{m} W_{m m}+a_{m} \hat{\pi}_{n n}\left(P_{m}-P_{l}\right) \\
& \mathcal{A}_{55}=\mathcal{A}_{66}=-\operatorname{diag}\left\{U_{m 1}, \cdots, U_{m(m-1)}, U_{m(m+1)}, \cdots, U_{m s}\right\} \\
& \mathcal{C}_{15}=\mathcal{C}_{26}=\left[\begin{array}{ll}
P_{m}-P_{l} & 0
\end{array}\right]^{T}
\end{aligned}
$$

The observer gain matrices are computed by $L_{i, m}=P_{m}^{-1} Y_{i, m}$.

Proof. (Case I): $i \in I_{i, k n}$ and $I_{i, k n}=\mathcal{S}$.

According to the partition of TRs, letting $\sum_{n=1, n \neq m}^{s} \pi_{m n}=-\pi_{m m}$. Therefore, $\sum_{n=1}^{s} \pi_{m n}(h) P_{j}$ also is

$$
\begin{aligned}
\sum_{n=1}^{s} \pi_{m n} P_{n} & =\sum_{n=1}^{s} \pi_{m n} P_{j}+\sum_{n=1}^{s} \Delta \pi_{m n} P_{n} \\
& =\sum_{n=1}^{s} \pi_{m n} P_{n}+\sum_{n=1, n \neq m}^{s} \Delta \pi_{m n} P_{n}+\Delta \pi_{m m} P_{m} \\
& =\sum_{n=1}^{s} \pi_{m n} P_{n}+\sum_{n=1, n \neq m}^{s} \Delta \pi_{m n}\left(P_{n}-P_{m}\right) \\
& =\sum_{n=1}^{s} \pi_{m n} P_{n}+\sum_{n=1, n \neq m}^{s}\left[\frac{1}{2} \Delta \pi_{m n}\left(P_{n}-P_{m}\right)+\frac{1}{2} \Delta \pi_{m n}\left(P_{n}-P_{m}\right)\right] \\
& \leq \sum_{n=1}^{s} \pi_{m n} P_{n}+\sum_{n=1, n \neq m}^{s}\left[\frac{\delta_{i j}^{2}}{4} U_{m n}+\left(P_{n}-P_{m}\right) U_{m n}^{-1}\left(P_{n}-P_{m}\right)\right]
\end{aligned}
$$

By using Schur's complement theory, it seems known that (26) supports Theorem 2 holds in this case. 
(Case II): Since $m \in I_{m, u k}$, and $I_{m, k n}=\varnothing$, while there exists a $n \neq m$ such that $n \in I_{n, k n}$. Here, $\pi_{m m}$ is estimated by $a_{m} \pi_{n n}$. Denoting $\lambda_{m, k n} \triangleq \pi_{m m}$. Therefore $\sum_{n=1}^{s} \pi_{m n} P_{n}$ can be written as

$$
\begin{aligned}
\sum_{n=1}^{s} \pi_{m n} P_{n} & =\pi_{m m} P_{m}+\sum_{n \in I_{m, u k n}} \pi_{m n} P_{n} \\
& =\pi_{m m} P_{m}-\lambda_{m, k} \sum_{n \in I_{m, u k n}} \frac{\pi_{m n}}{-\lambda_{m, k}} P_{n}
\end{aligned}
$$

Noting that $\sum_{n \in I_{m, u k n}} \pi_{m n}=-\pi_{m m}=-\lambda_{m, k}>0$. For $\forall l \in I_{m, u k n}$, it satisfies that

$$
\begin{aligned}
\breve{\Phi}_{i, m}+\operatorname{diag}\left\{\sum_{n=1}^{s} \pi_{m n} P_{n}, 0, \sum_{n=1}^{s} \pi_{m n} P_{n}, 0\right\} & =\sum_{n \in I_{m, u k n}} \frac{\pi_{m n}}{-\lambda_{m, k}}\left[\breve{\Phi}_{i, m}+\operatorname{diag}\left\{\pi_{m m}\left(P_{m}-P_{l}\right), 0, \pi_{m m}\left(P_{m}-P_{l}\right), 0\right\}\right] \\
& =\breve{\Phi}_{i, m}+\operatorname{diag}\left\{\pi_{m m}\left(P_{m}-P_{l}\right), 0, \pi_{m m}\left(P_{m}-P_{l}\right), 0\right\} \\
& =\breve{\Phi}_{i, m}+\operatorname{diag}\left\{a_{m} \pi_{n n}\left(P_{m}-P_{l}\right), 0, a_{m} \pi_{n n}\left(P_{m}-P_{l}\right), 0\right\}
\end{aligned}
$$

In the above formula (30), it has

$$
a_{m} \pi_{n n}\left(P_{m}-P_{l}\right)=a_{m} \hat{\pi}_{n n}\left(P_{m}-P_{l}\right)+a_{m} \Delta \hat{\pi}_{n n}\left(P_{m}-P_{l}\right)
$$

Then, with the help of Lemma 1 and for any $W_{m m}>0$, it can be obtained that

$$
\begin{aligned}
\Delta \hat{\pi}_{n n}\left(P_{m}-P_{l}\right) & =\left[\frac{1}{2} \Delta \hat{\pi}_{n n}\left(P_{m}-P_{l}\right)+\frac{1}{2} \Delta \hat{\pi}_{n n}\left(P_{m}-P_{l}\right)\right] \\
& \leq\left[\frac{\left(\delta_{n n}\right)^{2}}{4} W_{m m}+\left(P_{m}-P_{l}\right) W_{m m}^{-1}\left(P_{m}-P_{l}\right)^{T}\right]
\end{aligned}
$$

In combination with (28)-(32), by using Schur complement theory, Theorem 2 holds from (27) in this case.

Remark 7. When analyzing the conditions in Theorem 2, this is an important issue that how to determine $a_{i}$. Therefore, proposing the maximum optimization problem to settle this problem, that is

$$
\max . \sum a_{i}
$$

subject to $P_{i}, \ldots, \Omega_{m}$ in (26), (27).

Therefore, the $H_{\infty}$ performance is ensured with unknown TRs.

\subsection{Reachability of Sliding Surface}

In the section, to guarantee the accessibility of the sliding surface $s(t)=0$, it will be confirmed that the control scheme proposed will make the estimated state to the predesigned sliding surface in the limited range of time.

Theorem 3. Assuming that the conditions in Theorem 2 are solvable and (18) is proposed. By the fuzzy SMC law synthesized as follows, the state trajectories of (16) will be driven onto the sliding surface $s(t)=0$ in the limited range of time:

$$
u(t)=\sum_{i=1}^{r} h_{i}(\hat{\theta}) K_{i, m} \hat{x}(t)-(\rho(t)+\delta) \operatorname{sgn}(s(t))
$$

in which $\delta$ is a small positive tuning scalar, and

$$
\rho(t)=\max _{m \in \mathcal{S}} \sum_{i=1}^{r} h_{i}(\hat{\theta}) \lambda_{m}^{-1}\left\|\left(G B_{m}\right)\right\|^{-1}\left[\left\|G L_{i, m}\right\|\|\bar{y}(t)\|+\left\|G L_{i, m} C_{m}\right\|\|\hat{x}(t-\tau(t))\|\right]
$$


Proof. Choose Lyapunov function as follows:

$$
V(t)=\frac{1}{2} s^{T}(t) s(t)
$$

Then

$$
\begin{aligned}
\mathcal{L} V(t) & =s^{T}(t) \dot{s}(t) \\
& =s^{T}(t) \sum_{i=1}^{r} h_{i}(\hat{\theta}) G\left[-\lambda_{m} B_{m} K_{i m} \hat{x}(t)+\lambda_{m} B_{m} u(t)+L_{i m}(\bar{y}(t)-y(t-\tau(t)))\right] \\
& \leq|s(t)| \sum_{i}^{r} h_{i}(\hat{x}(t))\left[\left\|G L_{i m} C_{m}\right\|\|\hat{x}(t-\tau(t))\|+\left\|G L_{i m}\right\|\|\bar{y}(t)\|\right] \\
& -s^{T}(t) \sum_{i=1}^{r} h_{i}(\hat{x}(t)) \lambda_{m} G B_{m} K_{i, m} \hat{x}(t)+\lambda_{m} s^{T}(t) G B_{m} u(t)
\end{aligned}
$$

By substituting (33) into (35), we can obtain:

$$
\mathcal{L} V(t) \leq-\delta\|s(t)\|<0 \text { for } s(t) \neq 0
$$

Noting that $\|s(t)\| \leq|s(t)|$, then

$$
\mathcal{L} V(t) \leq-\gamma\|s(t)\| \leq-\sqrt{2} \gamma V^{1 / 2}(t)
$$

Now, consider the equation as follows:

$$
d V(t)=-\sqrt{2} \gamma V^{1 / 2}(t) d t
$$

where $V^{-1 / 2}(t) d V(t)=-\sqrt{2} \gamma d t$

from 0 to $t^{*}$, we integrate both sides it will yield

$$
2 \mathbb{E}\left[V^{1 / 2}\left(t^{*}\right)-V^{1 / 2}(0)\right]=-\sqrt{2} \gamma t^{*}
$$

from this, it is known that a constant $t^{*}=\sqrt{2 V(0)} / \gamma$ exists such that $\mathbb{E} V^{1 / 2}\left(t^{*}\right)=0$ (for all $t \geq t^{*}$, that is $\mathbb{E} s(t)=0$ ). In the case where $d V(t)<-\sqrt{2} \gamma V^{1 / 2}(t)$, because of monotonicity, $t^{*}$ is much smaller. As a result, the reachability is almost guaranteed in the limited range of time. The theorem is completely proved.

Remark 8. Compared with the traditional Markov jump system with completely known transfer rate, this study gives a stochastic stability criterion with completely unknown transfer rate of a certain mode, which extends the theoretical depth of this kind of system.

Remark 9. Theorem 3 not only illustrates the finite time reachability of sliding mode, but also proves the upper bound of the arrival time.

\section{Numerical Example}

Considering the dynamic equation of the single-link robot arm model as mentioned before

$$
\ddot{\theta}(t)=-\frac{M g L}{J} \sin (\theta(t))-\frac{D(t)}{J} \dot{\theta}(t)+\frac{1}{J} u(t)
$$

In detail, $g=9.81$ and $L=0.5$, the time invariant $D(t)=D_{0}=2 . M$ and $J$ have three different modes as shown in Table 2. Following the fuzzy approach in Part II, the state-space can be described as follows: 
Table 2. Parameters for $M$ and $J$ of Different Modes.

\begin{tabular}{ccc}
\hline Mode $\boldsymbol{m}$ & Parameter $\boldsymbol{M}$ & Parameter $\boldsymbol{J}$ \\
\hline 1 & 1 & 1 \\
2 & 1.5 & 2 \\
3 & 2 & 2.5 \\
\hline
\end{tabular}

Plant Rule 1: IF $x_{1}(t)$ is "about 0 rad", THEN

$$
\left\{\begin{array}{l}
\dot{x}(t)=A_{1, m} x(t)+B_{m} u(t) \\
y(t)=C_{m} x(t)
\end{array}\right.
$$

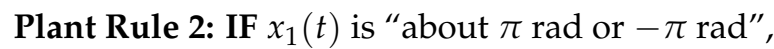
THEN

$$
\left\{\begin{array}{l}
\dot{x}(t)=A_{2, m} x(t)+B_{m} u(t) \\
y(t)=C_{m} x(t)
\end{array}\right.
$$

where $x(t)=\left[\begin{array}{ll}x_{1}^{T}(t) & x_{2}^{T}(t)\end{array}\right]^{T}$, and

$$
\begin{gathered}
A_{2,1}=\left[\begin{array}{cc}
0 & 1 \\
-\beta g L & -D_{0}
\end{array}\right], A_{2,2}=\left[\begin{array}{cc}
0 & 1 \\
-0.75 \beta g L & -0.5 D_{0}
\end{array}\right], A_{2,3}=\left[\begin{array}{cc}
0 & 1 \\
-0.8 \beta g L & -0.4 D_{0}
\end{array}\right] \\
B_{1}=\left[\begin{array}{l}
0 \\
1
\end{array}\right], B_{2}=\left[\begin{array}{c}
0 \\
0.5
\end{array}\right], B_{3}=\left[\begin{array}{c}
0 \\
0.4
\end{array}\right], C_{1}=C_{2}=C_{3}=\left[\begin{array}{ll}
1 & 1
\end{array}\right]
\end{gathered}
$$

First, let us check the SMC theory with fully known TR information, and the related TR matrix of the three operation modes is given by:

$$
\left[\begin{array}{ccc}
-2.5 & 0.8 & 1.7 \\
1.8 & -3.5 & 1.7 \\
0.6 & 0.7 & -1.3
\end{array}\right]
$$

In this way, we can check the effectiveness of the proposed results based on Theorem 1. Suppose $T=0.1 s$ and $\bar{d}_{m}=0.2$. In addition, letting the gain matrices $K_{1 m}=\left[\begin{array}{ll}-2 & -3\end{array}\right]$, $K_{2 m}=\left[\begin{array}{ll}-12 & -6\end{array}\right], \lambda_{m}=2, a_{i m}=0.1,(i=1,2 ; m=1,2,3), \rho=0.1, G=\left[\begin{array}{ll}1 & 0\end{array}\right]$ and $\gamma=3.5$. By solving the condition in (22), it obtains the following feasible solutions:

$$
\begin{gathered}
P_{1}=\left[\begin{array}{ll}
8.7998 & 1.9976 \\
1.9976 & 1.4892
\end{array}\right], P_{2}=\left[\begin{array}{ll}
7.8970 & 1.7822 \\
1.7822 & 1.8142
\end{array}\right], P_{3}=\left[\begin{array}{ll}
8.1524 & 1.5095 \\
1.5095 & 2.0841
\end{array}\right], \\
Q_{1}=\left[\begin{array}{cc}
11.8241 & 2.5154 \\
2.5154 & 1.4871
\end{array}\right], Q_{2}=\left[\begin{array}{ll}
1.4191 & 0.6498 \\
0.6498 & 2.1203
\end{array}\right], R_{1}=\left[\begin{array}{ll}
6.4342 & 1.4680 \\
1.4680 & 1.5033
\end{array}\right], \\
R_{2}=\left[\begin{array}{ll}
2.0690 & 0.0436 \\
0.0436 & 0.3314
\end{array}\right], Y_{11}=\left[\begin{array}{l}
0.1431 \\
0.0938
\end{array}\right], Y_{12}=\left[\begin{array}{l}
0.1390 \\
0.1162
\end{array}\right], Y_{13}=\left[\begin{array}{l}
0.2078 \\
0.1707
\end{array}\right], \\
Y_{21}=\left[\begin{array}{l}
0.3133 \\
0.1300
\end{array}\right], Y_{22}=\left[\begin{array}{l}
0.1136 \\
0.0970
\end{array}\right], Y_{23}=\left[\begin{array}{l}
0.2049 \\
0.1844
\end{array}\right] \\
\Omega_{1}=0.1125, \Omega_{2}=0.1006, \Omega_{3}=0.0642
\end{gathered}
$$


Therefore, the gain matrices of the observer are computed as

$$
\begin{aligned}
& L_{11}=\left[\begin{array}{ll}
0.0028 & 0.0592
\end{array}\right]^{T}, L_{12}=\left[\begin{array}{ll}
0.0040 & 0.0601
\end{array}\right]^{T}, L_{13}=\left[\begin{array}{ll}
0.0119 & 0.0733
\end{array}\right]^{T} \\
& L_{21}=\left[\begin{array}{ll}
0.0227 & 0.0568
\end{array}\right]^{T}, L_{22}=\left[\begin{array}{ll}
0.0030 & 0.0505
\end{array}\right]^{T}, L_{23}=\left[\begin{array}{ll}
0.0101 & 0.0811
\end{array}\right]^{T}
\end{aligned}
$$

Next, let us consider the case that the TR information is deficient, for instance, the related TR matrix of the three operation modes is given by:

$$
\left[\begin{array}{ccc}
-2.5+\hat{\Delta}_{11} & 0.8+\hat{\Delta}_{12} & 1.7+\hat{\Delta}_{13} \\
1.8+\hat{\Delta}_{21} & -3.5+\hat{\Delta}_{22} & 1.7+\hat{\Delta}_{23} \\
? & ? & ?
\end{array}\right]
$$

Here, we can estimate $\pi_{33}$ by $\pi_{33}=a_{3} \pi_{11}$. By selecting $a_{3}=1, \hat{\Delta} \pi_{m n} \leq \delta_{m n}=\left|0.1 \hat{\pi}_{m n}\right|$, and the other parameters are chosen as above. After solving the conditions in Theorem 2, it is easy to obtain:

$$
\begin{gathered}
P_{1}=\left[\begin{array}{ll}
7.6528 & 1.7503 \\
1.7503 & 1.4026
\end{array}\right], P_{2}=\left[\begin{array}{ll}
7.0124 & 1.5473 \\
1.5473 & 1.6671
\end{array}\right], P_{3}=\left[\begin{array}{ll}
7.4199 & 1.4446 \\
1.4446 & 1.9999
\end{array}\right], \\
Q_{1}=\left[\begin{array}{ll}
8.8393 & 2.0522 \\
2.0522 & 1.4767
\end{array}\right], Q_{2}=\left[\begin{array}{ll}
1.3527 & 0.6770 \\
0.6770 & 2.1394
\end{array}\right], R_{1}=\left[\begin{array}{ll}
4.5128 & 0.9448 \\
0.9448 & 1.3326
\end{array}\right], \\
R_{2}=\left[\begin{array}{ll}
1.9617 & 0.0627 \\
0.0627 & 0.3889
\end{array}\right], Y_{11}=\left[\begin{array}{l}
0.1482 \\
0.0974
\end{array}\right], Y_{12}=\left[\begin{array}{l}
0.1239 \\
0.1174
\end{array}\right], Y_{13}=\left[\begin{array}{l}
0.2063 \\
0.1862
\end{array}\right], \\
Y_{21}=\left[\begin{array}{l}
0.3290 \\
0.1490
\end{array}\right], Y_{22}=\left[\begin{array}{l}
0.0980 \\
0.1031
\end{array}\right], Y_{23}=\left[\begin{array}{l}
0.0768 \\
0.0730
\end{array}\right] \\
\Omega_{1}=0.1148, \Omega_{2}=0.0661, \Omega_{3}=0.0763
\end{gathered}
$$

Therefore, the gain matrices of the observer are computed as

$$
\begin{aligned}
& L_{11}=\left[\begin{array}{ll}
0.0049 & 0.0634
\end{array}\right]^{T}, L_{12}=\left[\begin{array}{ll}
0.0027 & 0.0679
\end{array}\right]^{T}, L_{13}=\left[\begin{array}{ll}
0.0113 & 0.0850
\end{array}\right]^{T}, \\
& L_{21}=\left[\begin{array}{ll}
0.0262 & 0.0736
\end{array}\right]^{T}, L_{22}=\left[\begin{array}{ll}
0.0004 & 0.0615
\end{array}\right]^{T}, L_{33}=\left[\begin{array}{ll}
0.0038 & 0.0337
\end{array}\right]^{T}
\end{aligned}
$$

Now, let us consider the maximum allowable $a_{3}$ for feasible solutions of the system with the above parameters by solving the optimal problems in Remark 7. For different $H_{\infty}$ attenuation levels $\gamma$ with fixed error tolerance $\rho=0.1$ and transmission delay $d_{m}=0.1$, we can see the maximum allowable $a_{3}$ for different attenuation levels $\gamma$ in Figure 1. From these results, it is easy to obtain that the proposed scheme can reduce the average transmission frequency while maintaining the control performance.

In addition, the state response of the overall closed-loop system with initial conditions $x(t)=[0.1 \pi-0.5]^{T}$ and $\hat{x}(t)=[00]^{T}$ is presented in the following Figures 2-4. Figure 2 depicts the state response of original system under control; Figure 3 plots the state response of observer system under control; the controller input is given in Figure 4. 


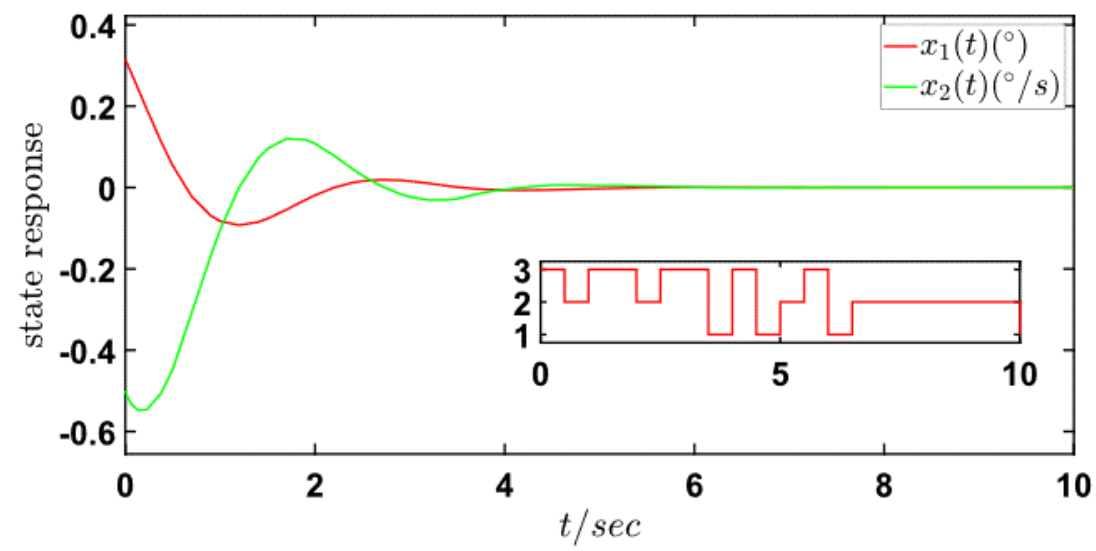

Figure 2. The state response of original system.

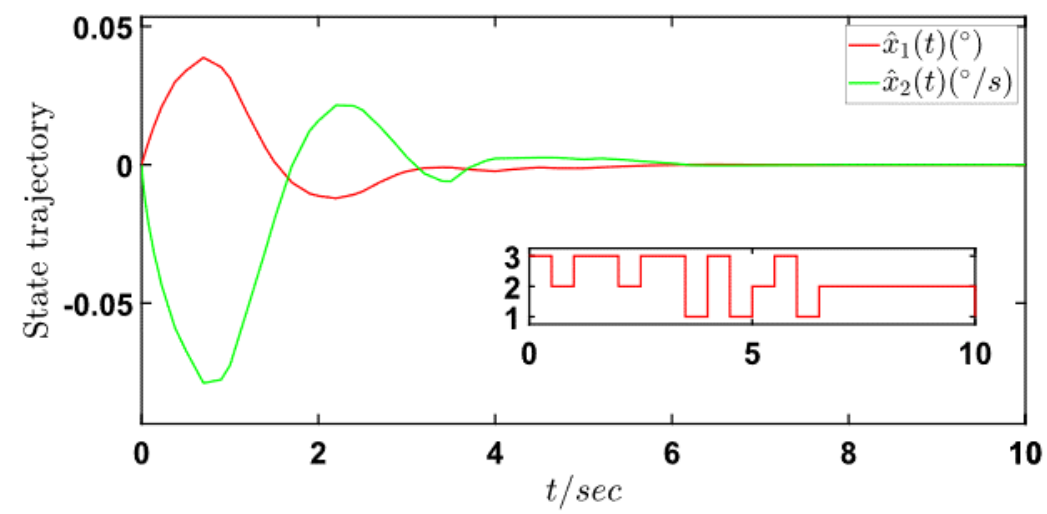

Figure 3. The state trajectories for the observer system.

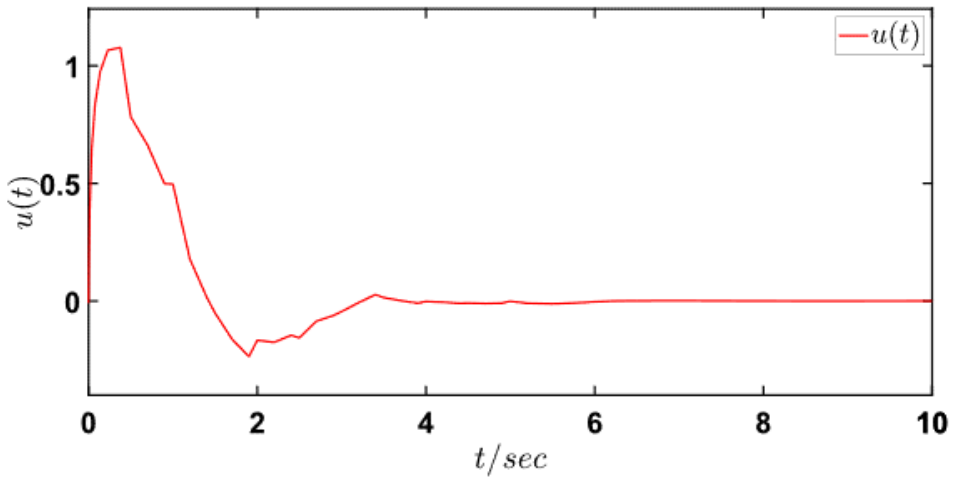

Figure 4. The control input.

Note that although the issue about sliding mode control based on observer for T-S model-based Markovian jump systems has been investigated in this paper, it still leaves much space for improvements. A future study should tackle new problems such as time delay and packet dropout.

Therefore, the design of state observer in this paper is implemented in the following way: First, select appropriate gain matrices $K_{i, m}$ such that $A_{i, m}+B_{m} K_{i, m}$ is Hurwitz. Second, obtain observer gain matrices $L_{i, m}$ by solving the inequalities in Theorem 1 or Theorem 2 . Third, set an event-generator based on the parameter obtained in the second step. Last, design the sliding mode controller proposed in (33). The diagram of overall implementation is presented in the following Figure 5. 


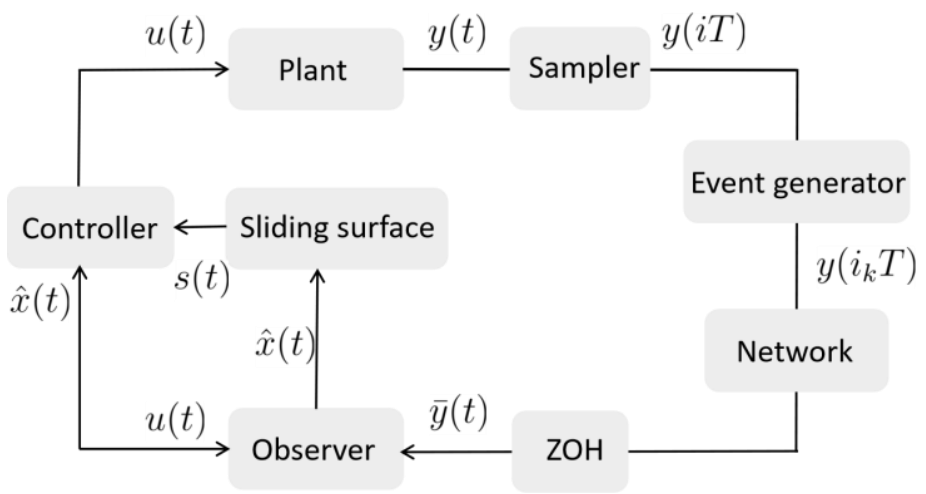

Figure 5. The diagram of overall closed-loop system.

Finally, in order to verify the advantage of the proposed method, a simulation study is conducted by comparing the system performance on original system based on the state observer without output time-delay, i.e., $\hat{y}(t-\tau(t))$ is changed by $\hat{y}(t)$. Taking the same parameters above, the simulation result is presented in Figure 6, from which it is seen that, compared with the system performance in Figure 2, much longer time is needed for the system to reach its steady state and the system stability is also affected to some extent. Therefore, proposing a time-delay Luenberger observer is a benefit for the system performance.

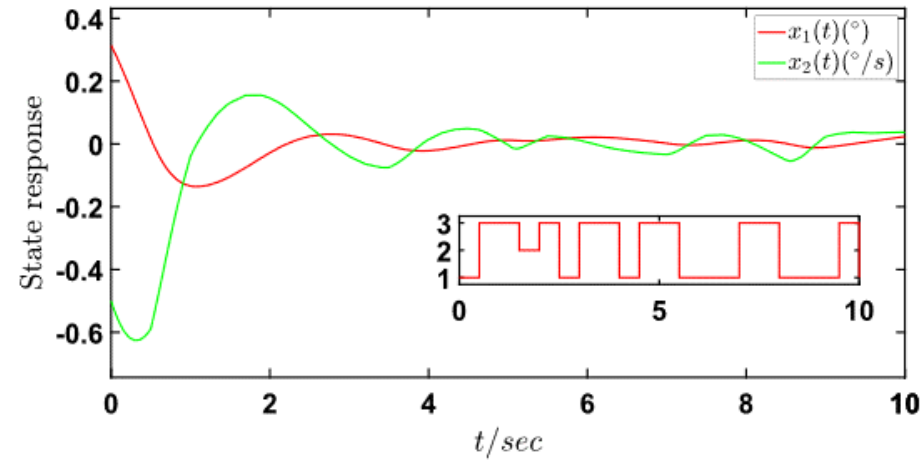

Figure 6. The state response of original system.

\section{Conclusions}

In this paper, the issue about sliding mode control based on observer for T-S modelbased Markovian jump systems was investigated. Firstly, it involved designing an eventtriggered based time-delay sliding mode observer, which can suppress the error and obtain good stability. On this basis, a novel integral sliding surface was proposed and the observer gain matrices can be computed in the design process. Then, according to stochastic stability theory, the $H_{\infty}$ performance of the sliding mode dynamics and the error dynamics were ensured in terms of LMI conditions. In addition, a fuzzy sliding mode controller was constructed to guarantee the finite-time reachability of the predefined sliding surface. Finally, numerical examples based on robotics were presented to verify the effectiveness of the proposed method.

Author Contributions: Conceptualization, C.Z., J.Q. and Z.W.; methodology, M.C., C.Z. and Z.W.; software, M.C., C.Z. and Z.W.; validation, M.C., Z.W. and Q.G.; formal analysis, M.C., J.Q. and Z.W.; investigation, M.C., and Z.W.; resources, M.C., Z.W. and Q.G.; data curation, M.C., C.Z. and J.Q.; writing—original draft preparation, M.C.; writing—review and editing, M.C., J.Q. and Z.W.; visualization, M.C. and C.Z.; supervision, J.Q., Z.W. and Q.G.; project administration, J.Q., Z.W. and Q.G.; funding acquisition, J.Q., Z.W. and Q.G. All authors have read and agreed to the published version of the manuscript. 
Funding: This research was funded by Alexander von Humboldt Foundation of Germany: Alexander von Humboldt Foundation of Germany and National Natural Science Foundation of China (No. 61903016).

Institutional Review Board Statement: Not applicable.

Informed Consent Statement: Not applicable.

Data Availability Statement: None.

Conflicts of Interest: The authors declare no conflict of interest.

\section{References}

1. Krasovskii, N.M.; Lidskii, E.A. Analytical design of controllers in systems with random attributes. Autom. Remote Control 1961, 22, 1021-2025.

2. Ugrinovskii, V.; Pota, H.R. Decentralized control of power systems via robust control of uncertain Markov jump parameter systems. Int. J. Control 2005, 78, 662-677. [CrossRef]

3. Andrieu, C.; Davy, M.; Doucet, A. Efficient particle filtering for jump Markov systems. Application to time-varying autoregressions. IEEE Trans. Signal Process. 2003, 51, 1762-1770. [CrossRef]

4. Wallace, V.L.; Rosenberg, R.S. Markovian models and numerical analysis of computer system behavior. In Proceedings of the Spring Joint Computer Conference, Boston, MA, USA, 26-28 April 1966; pp. 141-148. [CrossRef]

5. Ellis, R.S. Large deviations for the empirical measure of a Markov chain with an application to the multivariate empirical measure. Ann. Probab. 1988, 16, 1496-1508. [CrossRef]

6. Souza, C.E.D. Robust stability and stabilization of uncertain discrete-time Markovian jump linear systems. IEEE Trans. Autom. Control 2006, 51, 836-841. [CrossRef]

7. Gao, H.; Lam, J.; Xu, S.; Wang, C. Stabilization and Ho control of two-dimensional Markovian jump systems. IMA J. Math. Control Inf. 2004, 21, 377-392. [CrossRef]

8. Mao, X.; Yuan, C. Stochastic Differential Equations with Markovian Switching; Imperial College Press: London, UK, 2006. [CrossRef]

9. Wu, L.; Shi, P.; Gao, H.; Wang, C. Ho filtering for 2D Markovian jump systems. Automatica 2008, 44, 1849-1858. [CrossRef]

10. Xu, S.; Lam, J.; Mao, X. Delay-dependent $\mathrm{H} \infty$ control and filtering for uncertain Markovian jump systems with time-varying delays. IEEE Trans. Circuits Syst. I Regul. Pap. 2007, 54, 2070-2077. [CrossRef]

11. Fei, Z.; Gao, H.; Shi, P. New results on stabilization of Markovian jump systems with time delay. Automatica 2009, 45, 2300-2306. [CrossRef]

12. Zhang, L.; Huang, B.; Lam, J. Ho model reduction of Markovian jump linear systems. Syst. Control Lett. 2003, 50, 103-118. [CrossRef]

13. Jilkov, V.P.; Li, X.R. Online Bayesian estimation of transition probabilities for Markovian jump systems. IEEE Trans. Signal Process. 2004, 52, 1620-1630. [CrossRef]

14. Wu, Z.; Su, H.; Chu, J. Ho filtering for singular Markovian jump systems with time delay. Int. J. Robust Nonlinear Control 2010, 20, 939-957. [CrossRef]

15. Costa, O.L.V.; Guerra, S. Stationary filter for linear minimum mean square error estimator of discrete-time Markovian jump systems. IEEE Trans. Autom. Control 2002, 47, 1351-1356. [CrossRef]

16. Takagi, T.; Sugeno, M. Fuzzy identification of systems and its applications to modeling and control. IEEE Trans. Syst. Man Cybern. 1985, SMC-15, 116-132. [CrossRef]

17. Wang, Y.; Xia, Y.; Shen, H.; Zhou, P. SMC design for robust stabilization of nonlinear Markovian jump singular systems. IEEE Trans. Autom. Control 2018, 63, 219-224. [CrossRef]

18. Saravanakumar, R.; Ali, M.S.; Karimi, H.R. Robust $\mathrm{H} \infty$ control of uncertain stochastic Markovian jump systems with mixed time-varying delays. Int. J. Syst. Sci. 2017, 48, 862-872. [CrossRef]

19. Park, I.S.; Kwon, N.K.; Park, P. H $\infty$ control for Markovian jump fuzzy systems with partly unknown transition rates and input saturation. J. Frankl. Inst. 2018, 355, 2498-2514. [CrossRef]

20. Shen, H.; Li, F.; Yan, H.; Karimi, H.R.; Lam, H. Finite-time event-triggered Ho control for T-S fuzzy Markov jump systems. IEEE Trans. Fuzzy Syst. 2018, 26, 3122-3135. [CrossRef]

21. Dong, S.; Wu, Z.; Pan, Y.; Su, H.; Liu, Y. Hidden-Markov-model-based asynchronous filter design of nonlinear Markov jump systems in continuous-time domain. IEEE Trans. Cybern. 2019, 49, 2294-2304. [CrossRef]

22. Zhang, L.; Boukas, E.-K. Stability and stabilization of Markovian jump linear systems with partly unknown transition probabilities. Automatica 2009, 45, 463-468. [CrossRef]

23. Xiong, L.; Tian, J.; Liu, X. Stability analysis for neutral Markovian jump systems with partially unknown transition probabilities. J. Frankl. Inst. 2012, 349, 2193-2214. [CrossRef]

24. Kao, Y.; Xie, J.; Wang, C. Stabilization of singular Markovian jump systems with generally uncertain transition rates. IEEE Trans. Autom. Control 2014, 59, 2604-2610. [CrossRef]

25. Wei, Y.; Qiu, J.; Karimi, H.R. Quantized filtering for continuous-time Markovian jump systems with deficient mode information. Asian J. Control 2015, 17, 1914-1923. [CrossRef]

26. Liu, M.; Zhang, L.; Shi, P.; Zhao, Y. Sliding mode control of continuous-time Markovian jump systems with digital data transmission. Automatica 2017, 80, 200-209. [CrossRef] 
27. Zhang, H.; Wang, J.; Shi, Y. Robust Ho sliding-mode control for Markovian jump systems subject to intermittent observations and partially known transition probabilities. Syst. Control Lett. 2013, 62, 1114-1124. [CrossRef]

28. Song, J.; Niu, Y.; Zou, Y. Asynchronous sliding mode control of Markovian jump systems with time-varying delays and partly accessible mode detection probabilities. Automatica 2018, 93, 33-41. [CrossRef]

29. Li, H.; Shi, P.; Yao, D.; Wu, L. Observer-based adaptive sliding mode control for nonlinear Markovian jump systems. Automatica 2016, 64, 133-142. [CrossRef]

30. Liu, M.; Shi, P.; Zhang, L.; Zhao, X. Fault-tolerant control for nonlinear Markovian jump systems via proportional and derivative sliding mode observer technique. IEEE Trans. Circuits Syst. I Regul. Pap. 2011, 58, 2755-2764. [CrossRef]

31. Jiang, B.; Karimi, H.R.; Yang, S.; Gao, C.; Kao, Y. Observer-based adaptive sliding mode control for nonlinear stochastic Markov jump systems via T-S fuzzy modeling: Applications to robot arm model. IEEE Trans. Ind. Electron. 2021, 68, 466-477. [CrossRef]

32. Liu, Z.; Yu, J. Non-fragile observer-based adaptive control of uncertain nonlinear stochastic Markovian jump systems via sliding mode technique. Nonlinear Anal. Hybrid Syst. 2020, 38, 100931. [CrossRef]

33. Karimi, H.R. A sliding mode approach to $\mathrm{H} \infty$ synchronization of master-slave time-delay systems with Markovian jumping parameters and nonlinear uncertainties. J. Frankl. Inst. 2012, 349, 1480-1496. [CrossRef]

34. Zohrabi, N.; Reza Momeni, H.; Hossein Abolmasoumi, A. Sliding mode control of Markovian jump systems with partly unknown transition probabilities. IFAC Proc. Vol. 2013, 46, 947-952. [CrossRef]

35. Yue, D.; Tian, E.; Han, Q. A delay system method for designing event-triggered controllers of networked control systems IEEE Trans. Autom. Control 2013, 58, 475-481. [CrossRef]

36. Su, X.; Liu, X.; Shi, P.; Song, Y.-D. Sliding mode control of hybrid switched systems via an event-triggered mechanism. Automatica 2018, 90, 294-303. [CrossRef]

37. Peng, C.; Han, Q.; Yue, D. To transmit or not to transmit: A discrete event-triggered communication scheme for networked Takagi-Sugeno fuzzy systems. IEEE Trans. Fuzzy Syst. 2013, 21, 164-170. [CrossRef]

38. Behera, A.K.; Bandyopadhyay, B.; Yu, X. Periodic event-triggered sliding mode control. Automatica 2018, 96, 61-72. [CrossRef]

39. Dimarogonas, D.V.; Frazzoli, E.; Johansson, K.H. Distributed event-triggered control for multi-agent systems. IEEE Trans. Autom. Control 2012, 57, 1291-1297. [CrossRef]

40. Cheng, J.; Park, J.H.; Zhang, L.; Zhu, Y. An asynchronous operation approach to event-triggered control for fuzzy Markovian jump systems with general switching policies. IEEE Trans. Fuzzy Syst. 2018, 26, 6-18. [CrossRef]

41. Wang, L.; Wang, Z.; Wei, G.; Alsaadi, F.E. Finite-time state estimation for recurrent delayed neural networks with component-based event-triggering protocol. IEEE Trans. Neural Netw. Learn. Syst. 2018, 29, 1046-1057. [CrossRef] [PubMed]

42. Wu, L.; Gao, Y.; Liu, J.; Li, H. Event-triggered sliding mode control of stochastic systems via output feedback. Automatica 2017, 82, 79-92. [CrossRef]

43. Huai-Ning, W.; Kai-Yuan, C. Mode-independent robust stabilization for uncertain Markovian jump nonlinear systems via fuzzy control. IEEE Trans. Syst. Man Cybern. Part B 2006, 36, 509-519. [CrossRef] [PubMed]

44. Tanaka, K.; Kosaki, T. Design of a stable fuzzy controller for an articulated vehicle. IEEE Trans. Syst. Man Cybern. Part B 1997, 27, 552-558. [CrossRef] [PubMed]

45. Meyn, S.P.; Tweedie, R.L. Markov Chains and Stochastic Stability; Springer Science \& Business Media: Berlin/Heidelberg, Germany, 2012.

46. Boukas, K. Stabilization of stochastic nonlinear hybrid systems. Int. J. Innov. Comput. Inf. Control 2005, 1, 131-141. [CrossRef]

47. Xiong, J.; Lam, J. Robust $\mathrm{H}_{2}$ control of Markovian jump systems with uncertain switching probabilities. Int. J. Syst. Sci. 2009, 40, 255-265. [CrossRef]

48. Jiang, B.; Gao, C. Decentralized adaptive sliding mode control of large-scale semi-Markovian jump interconnected systems with dead-zone input. IEEE Trans. Autom. Control 2021. [CrossRef] 